\title{
Thomas Blanke Rechtstheorie und Propaganda Notizen zu Aufsätzen von E. Pašukanis aus der Stalin-Ära
}

\section{A. Einleitung}

Die nachstehenden, hier erscmals wieder veröffentlichen Aufsärze ${ }^{1}$ von Eugen Pašukanis aus den Jahren 1935 und 1936 sind, soweit ersichtlich, im Rahmen der vorliegenden Forschungsarbeiten über die Enrwicklung der sowjetischen Rechrstheorie zwischen der Oktoberrevolution und dem Stalinismus bislang nicht rezipiett worden"2.

Zur Zeit der Erstueröffentlichung dieser Texte war Pašukanis oder theoretische Kopf der sowjetischen Rechtswissenschafta'. Er war Vizekommissar der Jusciz, Mitglied der nach dem Parteitag von 1934 eingesetzten und bis 1936 tagenden Verfassungskommission (zusammen u. a. mit Bucharin), Leiter des Inscituts für Rechr der Akaderaie der Wissenschaften der UdSSR und gab die führende Rechiszeitschrift »Sowjetstaat (später "Sowjetstaac und Recht «) heraus. Seinen inrernationalen Ruf als einer der führenden marxistischen Rechestheoretiker hatte sein 1924 erschienenes Hauprwerk "Allgemeine Rechtslehre und Marxismus " begründer, welches bis 1927 drei Auflagen erlebre und 1929 ins Deursche übersetzr wurde." Obwohl er sowohl vor der Oktoberrevolution als auch in den Auseinanderseczungen der ersten Jahre der Sowjeturion keinerlei politisch bedeutsame Rolle gespieit hatte, avancierte er - bekannt geworden durch diese Schrift - schon in der ¿ weicen Hälfre der zoer Jahre zum Leiter der rechtswissenschaftlichen Sektion der Kommunistischen Akademie und zusammen mit Stučka zum Herausgeber der Zeitschrift "Revolurion des Reches*. Pasukanis gelang es in der stalinistischen Ara, seine einflußreiche Stellung bis zur Verabschiedung der Verlassung Ende 1936 nicht nur

\footnotetext{
1. Die Aulsäize sind zuerst enschienen in dem Organ der KPD im Exil, der in Basel herausgegebenen nundschau über Polizik, Wirschaft und Arbeiterbewegung* (Reprint bej Feltrinelli, Mailand 1967). Der Aulsatz ${ }^{2}$ Dic Lehre Lenins und Stalins.... wurde in Nr. 11 der Zeirschrift vom 28. 2. 1938 publizien, "Volkssıas Sowjeluniona in Nr. 25 vom 28. 5. 1936 und. Dic polirische und wircschaldiche Grundlage der Sowjeruniona in Nr. 29 vom 25. 6. 1936.

2 Norbert Reich crwähnt in seiner umfassenden Studie "Sozialismus und Zivilrecht". Frankfun 1972, diese Arbeiten cbensowenig wic in scinen sonstigen Unuersuchungen zu diesem Gegenstand: Einlcitung zu: Peu I. Sručka, ×Die revolutionärc Rollc von Recht und Staat $\alpha$, Frankfura/M. 1969, S. 7 ff; "Okrobcrevolution und Reche $\alpha$, KJ 197I, S. 133 ff.; "Mancistische Rechtsthcorie zovischen Revolucion und Sulinismus. Das Beispiel Pasukanisa, XJ 1972, S. 154 If.; "Marxistische und sozialisische Rechtscheorie*, FrankfurvM. 1972; auch in den Sudien van Wall Rosenbaum, "Zum Rechtsbegrifl bci Sručka und Pašukaris a, KJ 1972, S. 148 !l. und von Joachim Percls, "Zur politischen Veríassung des Sozialismus ", KJ 1972, S. 166 kf., finder sich kein Hinweis aul diose Aufsaize von Pašukanis, auch nichr bei Oskar Ncge, Thesen zur marxisuschen Rechustheorie, KJ 1973, S. 1 fl. (erweiterte Fassung in: H. Rouleuthner (Hrsg.). Probleme der manxistischen Rechtsheorie, Ffm r975. S. 10 fl.) und Henry Düx. Zur SubjektObjekı Dialekrik in der Rechis- und Staatsuheorie der DDR, KJ 1972, S. 349 ff.

I N. Reich, Marxiscische Rechtsthearie..., KJ 1971, S. Issi die nachstehenden Angaben über Pasukanis sind dieser Arbeit, der Studie "Sozialismus und Zivilrecble, a, 2. O. sowie der Untersuchung von Leonhard Schapiro, Die Geschichte der kommunissischen Partei der Sowjetunion, 0. O. (Fim), 196: crinommen.

4 Deutsche Ubersezzung untcr dem Titel, Allgemeinc Rechtslehre und Marxismus (mi einer Rezension von Karl Korsch), Nachdrack Flm 1966: ziticrt wird im (olgenden nach der 2. Aufl. von 1969.
} 
zu verteidigen, sondern sogar noch auszubauen, "Ebenso plötzlich wie sein Aufsrieg kommt auch sein Fall: Im Rahmen einer Hetzkampagne von Vyšinskij und anderen wird er als Volksschädling gebrandmarkt und ohne gerichtlichen Prozeß in den Kellern des NKWD erschossen. Fortan dürfen seine Werke nicht mehr zitiert werden. Sein Name wird aus den Annalen der Rechtstheorie getilgtes. Und dies, obwohl er selbse in seinen späteren, seit $1929^{6}$ esschienen Arbeiten, zunehmend deutlicher und schärfer von seinen frühen theoretischen Auffassungen vom almählichen Absterben von Recht und Staat im Übergang zur sozialistischen Gesellschaft abgerückt war. In einem im März 1936 publizierten Aufsatz ${ }^{7}$, der in der bisherigen Pašukanis-Forschung für sseine wohl letzte Außerung ${ }^{8}{ }^{8}$ gehalten wurde, geht er sogar soweit mit seiner urspränglichen rechtscheoretischen Position ins Gericht, daß er ihr wopportunistischen Unsinn "9, der wnichts mit dem Marxismus-Leninismus gemein habe 10 , bescheinig und auch seine Selbstkritik aus den Jahren $1930 / 31$ als "Heuchelei " abqualifiziert. In dieser Arbeit berichtet Pašukanis im übrigen von den Vorarbeiten für die neue Verfassung, die am r1. 6. 1936 vom Zencralen Vollzugsausschuß der Sowjetkongresse der Union angenommen und am 29. 11. 1936 von dem Allunions-Sowjetkongreß als "Scalinverfassung* mit geringfügigen Änderungen verabschieder wurde. Diese Verfassung, die bis in die Mitte der 7oer Jahre hinein Gültigkeit besaß, kommentiert (wenn dieser Ausdruck für eine gânzlich unkritische Lobpreisung gestattet ist) Pašukanis in dem dritcen hier wieder nachgedruckten Aufsatz aus der Rundschaua vom 25.6.1936 mil dem Titel: "Die politische und wirtschaftliche Grundlage der Sowjerunion «. Die beiden anderen Aufsätze "Die Lehre Lenins und Stalins vom Staate und die neue Erappe der Entwicklung der Sowjetdemokratie" vom 28. 5. 1935 und "Volksstaat Sowjetunion vom 28. 5. 1936 verfolgen überwiegend den Zweck, das deutschsprachige Publikum auf die Kernaussage der Stalinverfassung vom Ende der Ubergangsgesellschaft und der definitiven Errichtung des wsozialistischen Staates der Arbeiter und Bauern« (Arrikel 1) hinzuführen. Die Propagierung des ssozialistischen Staates« und eines mit der Okroberrevolution beginnenden nneue(n) sozialiscische(n) Sy-

, N. Reich, Marxistische Rechtstheorie ..., KJ 1972, S. 155.

Scit dem 20. Parteicag von 1956 wird zwar gelcgerslich wieder auf Pasukanis hingewiesen und eingestanden, daß die ihm zum Vorwurf gemachten Verbrechen konstruier gewesen scien. Allcrdings werden scine fruheren rechistheorctischen Auffassungen nach wie vor stercoryg als politisch gefährlich und falsch bezeichnet, vgl. Schapiro, a. a. O., S. 494, 609 und dic Nachweise bei Perels, Der stazilich yerordnete Sozialismus, in: H. Rouleuthner (Hrsg.), a. 2. O., S. 351,356 Anm. 63 sowic unten Tcil C Fn. 48; eine bemerkenswert posicive Bezugnahme auf P2sukanis' ,Allgemeine Rechtslchre und

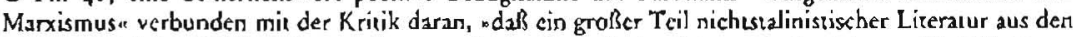
wissenschafelichen Bibliotheken entfernt werden mußee , finder sich bereits im Jahr 1948 bei Wolfgang Abendrort, damals Professor in der Sowjetischen Besatzungszone, in seinem Aulsatz: Die Justizreform in der Sowjeczone Deutschlands $\alpha$, Europa-Archiv, 1948, 9. Folge, S. I545, Anm. 68; vgl. hicrzu j. Pcrels, Eirleirung zu: W. Abendroch, Arbciterklasse, Srans und Verfassung (Hrsg.: J. Perels), S. 1) Anm. 28.

6 Der Zeitpunkt dieser erseten äftendichen Selbsckritik von Pašukanis steht olfensichtlich in Zusammenhang mil einer dramatischen politischen Kursverinderung seil der Plenarsitzung des ZK vom April 1929, auf der Stalin weinen offenen Angriff au! Bucharin unternahm und im besonderen Xußerungen cadclic, dic jencr in der Vergangenheir über das Abserben des Staales gemachı hare. Stalin betonce, dab nicht nur von einem Absterben des Srates keine Rede sein könne, sondern daß das Land nun in cine Periode des verstärkten Klassenk ampfes eintrete ... Angesichts dicscr höchsten Aucariür blicb Pašukanis nichts anderes übrig als zu widerroleu $\alpha$, Schapiro, z. z. O., S. 492.

7 Sraat und Recht im Sozialismus, in: Sowjetsuat 1936, Nr. S. $_{3}$ If, zit. bei N. Reich, Manxistische Rechtstheorie..., a. a. O. Fn. 3s (unter Hinwsis auf cine englische Ubersetzung) und bei $N$. Reich, Sozialismus und Zivilrecht, a. a. O. Fn. 676 .

8 N. Reich, Marxiscische Rechestheorie..., a. a. O. S. 162.

9 Zit. nach N. Reich, Marxist. Rechescheorie ..., s. a. O. S. 162

10 Zil. nach N. Reich, Sozialismus und Zivilrecht, 2. a. O. S. 257.

1 Zir. nach N. Reich, eld. 
stems des Rechrsa'2 stellt aber entsprechend der ursprünglichen Rechtstheorie von Pašukanis, nach der es in nachbürgerlichen Gesellschaften nur fortexistierendes bürgerliches Recht geben kann oder gar kein Recht (sondem ailein technische Regeln), eine theorerische Contradictio in adjecto dar: Eine historische und logische Unmöglichkeit. Indem Pašukanis diese Kehrtwende nicht nur vollzieht, sondern in der Auseinandersetzung, ja Abrechnung mit seinen früheren Positionen polemische Selbstkricik übt, liefert er der Anfang 1937 einseczenden offiziellen Verfemung seiner Lehre und der Pogromhetze gegen seine Person's auch noch die "wissenschaftlichen" Stichworte.

Wenn wir diese drei Aufsäcze hiemit erneut publizieren, so erfolgt dies nicht in der Absicht, den Überblick über die Publikationen dieses neben Stučka bedeutendsten marxistischen Rechtscheorecikers der frühen Sowjetunion zu vervollständigen - dafür sind die hier wieder zugänglich gemachten Texse für die rechtstheorerische Diskussion zu unergiebig. Auch ist die wissenschaftliche wie policische Biographie von Pašukanis, seine Wende zum Stalinismus bekannt und war bereits Gegenstand der Uncersuchung von Norber Reich, nMarxistische Rechtstheorie zwischen Revolucion und Stalinismus. Das Beispiel Paschukanisa in Heft $2 / 1972$ der "Kritischen Justiz *. Die Wiederveröffentlichung dieser späten, vermuzlich letzten Arbeiten von Pašukanis im Kontext der themasischen Schwerpunkrserzung dieses Heftes erfolgr in erster Linie aus dem Interesse einer exemplarischen Darstellung, Analyse und Kritik solcher Schriften, die im Gegensatz zu den früheren, theorecisch reflektierren Srudien von Pašukanis den offiziellen Mustern der staliniscischen Sprachregeiungen voll aufsitzen. Was, so ist zu fragen, kann aus solchen Texten unmittelbar, ohne detaillierte historische Zusatzinformationen erfahren und gelernt werden? Was kann aus ihnen erschlossen werden über spezifische politische und soziale Problemlagen und Auseinanderserzungen? Welche Funkrion kornmt diesen stalinistischen Sprachregelungen zu, was läßt sich über den Charakrer und die Mechanismen eines politischen Systems, welches Politik und wissenschaftliche Reflexion in dieser Weise öffentlich darstellt und allein diesen Modus öffenclicher Kommunikation zuläßt, aussagen? Damit wird insgesamt die Frage nach der spezifischen, auch juristischen Reflexionsweise des sowjetischen Gesellschaftssystems in der Epoche des Scalinismus gestellt - ein Problem, welches nach wie vor von aktueller Bedeurung ist für das Verständnis der sozialen Scrukruren und Herrschaftsmechanismen der sich als sozialistisch etiketrierenden Länder. '4 Diesen Problemen, die auch die Frage nach dem spezifisch stalinistischen Rechtsverständnis und die Untersuchung der etwaigen Kontinuität bzw. Diskontinuitär der rechtstheoretischen Position von Pašukanis umfassen, wird im Anschluß an die Wiederveröffentlichung der nachscehenden Aufsätze nachgegangen werden.

12 Zit. nach N. Reich, Marxistische Rechischeoric ..., a. a. O. S. 161 aus dem van ihm behandelecn Aufsarz von Pašukanis aus dem jahr 1936 (vgl. Fn. 6).

1) Die offiziclle Abrechnung*, so schreibr N. Reich, Sozialismus und Zivilrecht, a.a.O. S. 257, smit dim zum . Volksschädling, erklärnen und liquidicnen. Pašukanis beginnt mit einem Arrikel von Judin in der - Prawdar vom 20.1, 1937. Es folgen dic Thesen der Milarbeiter des Justizkommissarials, Angriffe von Vyšinski) und Orlon'skej, Selbstkriviken von Brarus' und Amfiteatrova, Bereivs 1936 hatte Vyšinskij von Pašukanis die Leirung des Insiruts für Recht der Akademic der Wissenschatten der UdSSR sowie die Redakcion der Zeischrifi "Sowjerstast a ibernommen, vgl. N. Reich, Sozialismus und Zivilrechu, 2. a. O. S. 256 , sowie Schapiro, a. 2. O. S. 43$\}$ ff.

14 Vgl. hierzu unter dem Aspekt der Forrgeltung bzw. Veränderung des staliniscischen Rechrsverständnisses J. Perels, Der scaatich verordncte Sozialismus, 2. a. O. S. 338 ff., insbes. S. $34 \mathrm{C}$ bis 344. 


\author{
DIE LEHRE LENINS UND STALINS \\ VOM STAATE UND DIE NEUE ETAPPE DER ENTWICKLUNG DER \\ SOWJETDEMOKRATIE [1935] \\ Von E. Pascbukanis (Moskan)
}

Der vom VII. Sowjetkongreß beschlossene Schritt in der weiteren Entfaltung der Sowjerdemokrarie folgr aus den Grundithesen der marxiscisch-leniniscischen Lehre vom Staate und von der Diktatur des Proletariats.

Die sewigen $\alpha$ und unerschütterlichen Grundlagen der Gesellschafr, die die Bourgeoisie in ihrer fühen Jugend verkündete, als sie als Vertecer des aligemeinen Fortschrittes auftrat, waren schnell gerostet und von der Bourgeoisie selbst weggeschoben worden. Die Angst vor dem Proletariat, das mit seinen Klassenforderungen auf dem Kampfplatz erscheint, veranlaßt die Bourgeoisie, bei den Kräften der Vergangenheit Hilfe zu suchen: sie schafft die Demokratie ab, verzichter auf großartige Losungen. Die Ekstase der bürgerlichen Revolutionen ist, wie Marx sagıe, von kurzer Dauer, sie geht vorbei und räumr den Platz der rückläufigen Bewegung.

Die proletarische Revolution behauptet nicht, daß die von ihr geschaffenen politischen Formen ewig dauern werden. Sie macht es sich nicht zur Aufgabe, eine unbewegliche, unerschürterliche Verfassung aufzustellen. Die Sowjerverfassung enthält nichts Statisches, Versteinertes und war gerade dadurch stets lebendig und wirksam, daß sie in allen Entwicklungsetappen ndie Diktatur des Proletariats zur Unterdrückung der Bourgeoisie, zur Aufhebung der Ausbeutung des Menschen durch Menschen und zur Verwirklichung des Kommunismus garantieren mußte, der die Spaltung der Gesellschaft in Klassen und den Sraat selbst aufhebr a (aus der Verfassung der RSFSR).

Die Theorie der Grundlage des Sowjetstaates wurde lange vor der Okcoberrevolution, schon während der Kämpfe Lenins gegen die Narodnikj, gegen Struwe, gegen die Okonomisten und Menschewiki ausgearbeitet. In diesen Kämplen hat Lenin die Grundchesen Marx' fortentwickelt und die Lehre von der Rolle des russischen Proletariats in der bevorstehenden revolutionären Epoche geschmieder, die Lehre von der Hegemonie des Proletariats, von der Bedeutung der bewußten Vorhut, über die Bedeurung der Ausnürzung der Staatsgewalt durch die revolutionäre Klasse.

Am Vorabend des Krieges 1914/18 waren die Zentristen und Sozialpatrioten aut die Anerkennung der Bedeurung des bürgerlichen Scaates als eine über den Klassen stehende gemeinnützige Institution eingegangen. In der Diskussion mit Pannekoek im Jahre 1912 hat Kaursky die Losung von der Zertrümmerung der bürgerlichen Staatsmaschine bereits für anarchistisch erklärr. Aut dieser wtheoretischen * Grundlage fanden sich die offenen Sozialannexionisten und Anhänger der sVereidigung des bürgerlichen Vaterlandes", wie auch die Pazifisten, die nach einer »demokratischen Welc seutzren, zusammen.

Zu dieser Zeit besaß die bolschewistische Partei bereits die Enfahnungen der Revolution 1905, die Lenin durch den Schmelzofen seiner Analyse getrieben hat. Die Diskussion mir den Menschewiki über die Teilnahme an der revolucionären Regierung und über die revolutionär-demokratische Diktacur des Prolecariats und

1. Dic Quellen für die Erstveröffentlichung sind aufgefführt in der Fn. I der Einlcitung, S. 401. (Red. KJ) 
der Bauexnschaft rollte die Frage auf, ob das Proletariat als Hegemon der bürgerlich-demokratischen Revolution mit der Perspektive ihres Hinüberwachsens in die sozialiscische Revolution, den Staztsapparat ausnüczen soll.

Während die österreichischen Sozialdemokraten ihre opportunistische "Theorie* über die rationale Frage ausarbeiteten, die die Erhaltung der bestehenden Landesgrenzen und reaktionären imperialistischen Staaten begründen sollte, haben Lenin und Stalin das proletarische, revolutionäre Programm in der nationalen Frage niedergelegt und kündigten dem "ökonomischen Imperialismus $\propto$ der Luxemburgisten den Kampf an, die sich sowohl von der nationalen Frage als auch von der Frage der Staatsgrenzen drüicken wollten, indem sie das Selbstbescimmungsrecht der Völker über Bord warfen.

In diesen Kämpfen wurde die Waffe geschmiedet, mir deren Hilfe die Partei nach der Oktoberrevolution die Vereinigung der Völker des ehemaligen Zarenreiches in einen festen, unerschütterlichen Bund, in der Union der Sozialistischen Sowjetrepubliken vollzog.

Wiederholt wies Lenin auf die Bedeurung hin, die die Revolucion von 1905 für die praktische und theorerische Vorbereitung des Kampfes um die Diktarur des Proletariats besaß. Nach der Oktoberrevolution schrieb er, daß ndie grundlegenden Fragen (Sowjermachc und Dikcatur des Proletariats), die gegeawärtig die Aufmerksamkeit der klassenbewußren Arbeiter in der ganzen Welr fesseln, prakrisch bereits Ende tgos gestellt worden sind, *

Die These, daß die Diktatur des Prolecariats die Vorausseczung der Entstehung eines neuen historischen Typus der Demokratie, der Demokratie für die Werktätigen darstellt, war Gegenstand hefriger Angriffe und Verleumdungen.

"Die Dikratur im allgemeinen $\alpha$ widerspricht der Demokratie im allgemeinen - das ist die Einstellung der sozialdemokratischen Theoreriker. Wenn das Proletariat seinen Klassenfeinden die Rechre und demokratischen Garantien entziehr, kann es keine Demokrarie für sich, keine Demokratie für die Werkeätigen bewahren, - so sagten diese Theoretiker. Diese verlogenen Behauprungen der Kautsky, Renner u. a. wurden vom Leben selbst widerlegt. Sie mögen uns ein noch so demokrarisches Land zeigen, in dem, wie bei uns, in so kurzer Zeit so gewaltige Massen von Werktätigen, insbesondere Bauern, an das bewußte politische Leben herangezogen wurden, sich aktiv an der Verwaltung des Staates bereiligen. Mögen sie uns noch einen Staat zeigen, der, wie der unsrige, sich raschen Schritzes von der alten Rückständigkeit der Frau enternce, ihre Isolierung vom öffentlichen Leben, wie wir, beseitigt härte.

In einem Jahrzehnt, von 1925 bis 1934 , ist die Akcivitär der Frauen bei den Wahlen fast auf das Niveau der Männer gestiegen (87 Prozent und 79 Prozent laut Angaben der RSFSR im Jahre 1934, während der Unterschied im Jahre 1925 noch 67 Prozent gegenüber 28 Prozent betrug.) Allein in den Sektionen der Sowjets waren im Jahre r 933 in der RSFSR I s 3000 Personen an die Staatsverwaltung herangezogen worden. Die Deputiertengruppen vereinigen etwa 350000 Menschen. Es wachsen Cheftumsbewegung und sozialistisches Vertrecungssystem, - diese vollkommen neuen Formen der Massenbereiligung an der Verwaitung und Kontrolle des $S_{\text {taats- }}$ apparaces.

Wo in der Welt ist der zentralen, lokalen, kollekrivwirtschafrlichen und Wandoresse ein ähnliches Arbeitsgebiet eingeräumt? Eine Armee von Arbeiter-Korrespondenten und Dorf-Korrespondenten, spezieller Brigaden führ bei uns den Kampf um eine neue, sozialistische Einscellung zur Arbeic und zur Produktion, um die Festigung und den Schurz des sozialistischen Eigentums, gegen Bürokraten, Faulenzer, Desorganisatoren der sozialistischen Produktion. 
Die vom VII. Sowjerkongreß angenommenen Beschlüsse beweisen ganz genau, daß gerade die Diktatur des Proletariats die Voraussetzungen einer wahren, ehrlichen Entscheidung mit Stimmenmehrheit ermöglichr, dadurch, daß sie die Wurzel des Kapicalismus ausmerzt, die skapitalistische Willensbescimmung und Motivierung des Willens" ausschließt. (Lenin).

Die Zerschlagung der kapiralistischen Elemente und der endgüluige Sieg des Kollektivwirtschaftssystems schufen die Grundlage für die Angleichung der Wahlrechre der Bavern an die der Arbeirerklasse, schufen die Voraussetzungen gleicher Wahlen in Stadt und Land.

Die Leninsche Lehre über den Staat hat sich in den Kämpfen gegen die kleinbürgerlich-opportunistischen Auffassungen, die manchmal selbst in die Reihen unserer Partei eindrangen, erhärtet.

Es gab bei uns in diesem Zusammenhang zwei Arten von Abweichungen, von denen Genosse Stalin auf dem XVI. Parreitag der KPdSU sprach. Nämlich den kleinbürgerlichen Liberalismus und den kleinbürgerlichen Radikalismus. Für den ersten ist die Verwischung des Klassenkampíes unter der Diktatur des Proletariars charakceristisch. Er stellt den Staat der Ubergangsperiode als "Ring « dar, der „die verschiedenen Klassen zusammenhält $\propto$. Bezeichnend für den kleinbürgerlichen Liberalismus sind des weirern die Kapitulation vor der Anarchie des Marktes, die Einstellung auf den Selbsclauf der Dinge, Skeptizismus gegenüber dem Stzatsplan, Wiederholung der überholten bürgerlichen und menschewistischen Behauptungen, daß wirtschaftliche Geseize nicht durch »adminustrative« Maßnahmen gemeistert werden können, usw.

Für den kleinbürgerlichen Radikalismus sind die Spielereien mit dem Anarchismus und Syndikalismus charakteriscrisch; die Angriffe auf das $\bowtie$ Auroritäre», d. h. einfach, sie sind dagegen, daß das Proletariat die von ihm eroberte Staatsgewalt gebrauche; ungeduldiger Wunsch, das Absterben des proletarischen Staates zu beschleunigen. Das nradikale Uberspringen einer Reihe von Erappen in dieser Frage und die Entstellung der Gedanken Marx' und Lenins über die besondere Natur des proletarischen Staares gehr Hand in Hand mit dem rechısopportuniscischen Geschrei über Schwächung der Dikratur des Prolecariass, Verminderung der Rolle des SowjerStaates in der Periode der Vernichtung der Klassen und der kapitalistischen Uberbleibsel in der Wircschaft und im Bewußssein der Menschen. Von diesen braven Helden des Automatismus sagte Genosse Stalin auf dem XVIII. Parteitag, daß sie die zur Losung gewordene These von unserem Vormarsch zur klassenlosen Gesellschaft als automatischen Prozeß aufgefaßc haben. Und sie fügten hinzu: wenn klassenlose Gesellschaft, dann kann man wohl den Klassenkampt muldern, die Diktacur des Proletariats abschwächen und mit dem Staate überhaupr Schluß machen, der in nächster Zeit sowieso absterben muß. Und sie gaben sich einem albernen Stolz hin, daß es bald keine Klassen mehr geben wird, d. h. also keinen Klassenkampf, d. h. also, es wird keine Sorgen und keine Aufregung mehr geben, daß man also die Waffen aus der Hand geben und sich auf die faule Seite legen könne, - in Erwartung der kJassenlosen Gesellschaft. « (Stalini Probleme des LeniDismus). ${ }^{2}$

An Stelle des Kamples gegen den geschlagenen, aber noch nicht restlos vernichteten Klassenfeind, der weiteren Entfaltung aller in der Sowjetdemokratie vorhandener Möglichkeiten, der Konzentrierung aller Mittel des $Z$ wanges und der Uberzeugung zum Schucze, zur Festigung und Entwicklung des sozialistischen Eigeneums, - dieser Grundlage unserer Gesellschaftsordnung, - setzen die Opportunisten angeneh-

2. Der Beginn des Zitass ist im Original nicht angegeben. (Red. $\mathrm{KJ}$ ) 
me Träume von der Lockerung und vom Verschwinden des proletarischen Staates. Dieses Gemisch von kleinbürgerlich-anarchistischen Stimmungen und schöngeistigem Liberalismus isc keine geringe Gefahr in der Epoche, in der die großen Aufgaben des zweiten Fünfjahrplanes gelöst werden, in der der von kapitalistischen Ländern umkreiste proletarische Staat alle Kräfte anspannt, um in der nahenden Auseinandersetzung den imperialistischen Räubern entsprechend zu begegnen.

Die erwähnten Änderungen in der Sowjetverfassung werfen ein helles Licht auf den theoretischen Xampf, den Genosse Stalin gegen die antileninistischen Behauprungen Sinowjews geführt hat, der im Lager der offenen Konterrevolution gelandet ist. Das war zur Zeit, als Sinowjew durch Verdrehung und Entstellung des Leninismus seine These von der «Diktacur der Partei« stützen wollte.

Diese These enthielt den Verzicht auf Entfaltung der Sowjerdemokratie, sie machte aus den Sowjets eine rein juristische Form. Die Diktatur der Klasse wurde angezweifelt, zwischen der Partei und den pareilosen Massen der Arbeiter und Bauern wurde ein Abgrund aufgerissen, die Rolle der Massenorganisationen, die die Weisungen der Parrei ins Leben einführen, wurde mißachcet.

Aus der Sinowjewschen These folgte, daß die Entfaltung der Sowjetdemokratie, daß die Möglichkeit für die Werktätigen, ihren Willen in der Staatsverwaltung zur Gelcung zu bringen, unter der Diktatur des Proletariats undenkbar sei. Es folgte aus der Sinowjewschen These zweitens, daß die Enffaltung der Demokratie die Abschwächung der Führerrolle der Partei zur Voraussetzung habe. Im großen und ganzen wiederholte diese sTheorie die weißgardistisch-menschewistische Lüge und Verleumdung des Sowjetstaates und unserer Partei. Ausgehend von dieser Sinowjewschen "Theorie weissagre Kamenew, daß die Belebung der Sowjers die "Verstopfung der unteren Stufen des Sowjetapparates durch kleinbürgerliche Elemente bedeute.

Die These von der "Diktatur der Parieiu konnte vom selben Sinowjew mit Leichtigkeir in die gemeine Verleumóng von einem Thermidor, von der Entarrung der Sowjetmacht umgewandelt werden.

Der troczkistisch-sinowjewistische Block entstand auf dem Boden der konterrevolurionären Kapitulanten-Theorie von der Unmöglichkeit des Aufbaus des Sozialismus in einem Lande. Eine andere wheorerische Basis bestand in der von Kaursky entlehnten Gegenüberstellung von Demokratie und Diktatur, der Leugnung dessen, daß die Epoche der Diktatur des Prolecariats die Epoche der Entfaltung einer neuen Demokratie höheren Typus ist, der proletarischen Sowjerdemokratie.

Es genügt, auf die Erklärung des Menschewiken Trotzki hinzuweisen, daß das System des Kriegskommunismus für die gesamte Ubergangsepoche charakteristisch sei; es genügt, an seine These zu erinnem, wonach das Proletariat einer kulturellen Schöpfung unfähig sei.

Die bürgerlichen und kleinbürgerlichen Politiker wenden sich nur insofern an die Massen, als sie sie nötig haben. Sie sind außerstande, die wirklichen Interessen dieser Massen zum Ausáruck zu bringen, daher bleibr ihnen nichts anderes übrig, als diese Massen zu betrügen, während sie sie in der Tiefe ihrer Seele verachten.

Unsere Partei, die den siegreichen Kampí um die Verwandlung des Rußland der Nep in ein sozialistisches Land fühn, hat sich bereits eine enge Verbundenheit mit den Millionenmassen, ihre Unterstützung, ihre aktive Beteiligung am Aufbau des Sozialismus gesichert.

„Die Diktatur des Proletariats - schrieb Genosse Stalin, - „besteht in leitenden Weisungen der Partei, plus der Durchführung dieser Weisungen durch die Massenorganisationen des Proletariats, plus ihrer Verwirklichung durch die Bevölkerung. « (Scalin: Probleme des Leninismus.) 
Die vom VI. Sowjetkongreß beschlossenen Anderungen in der Sowjerverfassung schaffen die Voraussetzungen für ein noch stärkeres Wachstum der Autorität und des Einflusses der Partei, für eine noch größere policische Akrivicär der Arbeiter und Kollektivbauern-Massen im Xampfe um die Vernichtung der Uberreste des Kapitalismus, für die Basis unserer Gesellschaftsordnung: das sozialistische, gesellschafrliche Eigentum.

Der Name Sowjerbürger, - Bürger des Landes, das als erstes in der Welt die Ketten des Kapitalismus zerbrach, - ist zum Stolz derer geworden, die ihn tragen und erfreut sich der Achrung und der Sympathie der Werktätigen aller Länder.

Durch den neuen Fortschritu der sozialistischen Demokratie ist dieser Name in der Achrung und Liebe des Weltprolecariats noch mehr gestiegen.

\section{VOLKSSTAAT SOWJETUNION [1936] \\ Von J. Paschukanis}

Die sozialistische Gesellschaft der Werküuigen ist in der Sowjetunion bereits aufgebaut.

Die Staarsmacht der Arbeiterklasse, die sich vor achrzebn Jahren die Aufgabe stellre, die Ausbeurung aus der Welt zu schaffen, hat dieses welngeschichrliche Werk vollbracht.

"Jetzt werden wir auf dem vom Gerümpel der Geschichte gesäuberten Wege«, sagte Lenin auf dem III. Sorvjetkongreß, »das starke, lichte Haus der sozialiscischen Gesellschaft aufbauen; es wird ein neuer in der Geschichce noch nie dagewesener Typus einer Staatsmacht geschaffen, die durch den Willen der Revolucion berufen isc, den Erdboden von jeglicher Ausbeutung, Gewalt und SkJaverei zu säubern.* Diese Aufgabe ist heute in der Hauptsache gelöst. Die Idee des Sozialismus ist in die Tat umgesetzt. Ein neuer, böberer Typus von Zivilisation wurde geschaffen, der den Ausblick auf einen gewaltigen, unaufbörlichen Aufschwung der materiellen und geistigen Kultur eröffnet. Das Land, das keine Krisen kennt, das Land, wo es keine Arbeirslosigkeit gibt, das Land, das mic der nationalen Unterdrückung aufgeräumt hat, das Land, das die völlige Gleichberechrigung der Frau genvährleistet hat, das Land, wo jeder Werkrätige des morgigen Tages gewiß isr und weiß, daß dem weiteren Aufschwung des öffentlichen und privaren Wohlstandes keine Grenzen gesetzr sind, dieses Land ist die Sowjetunion, der Staat der freien Arbeitsleute in Stadt und Land.

In der Sowjetunion sind die Klassen in der Hauptsache aufgeboben, denn das Privateigentum an Produktionsmitteln und die Möglichkeit der kapitalistischen Ausbeulung sind aufgehoben. Das Bündnis der Arbeiterklasse und der Bauernschaft ist in eine neue Phase eingetreten, denn sein Hauptziel - die Umwandlung der gestrigen kleinen Warenproduzenten in aktive Erbauer der sozialisuschen Gesellschaft - ist erreicht. Die Konsolidierung des Kollekrivwirrschaftssystems, der Sieg des gesellschaftlichen sozialiscischen Eigencums in Stadt und Laod bewirken ein langsames Verwischen der Klassenunterschiede zwischen Prolecariat und Bauemschaft. Die Einbürgerung der Maschine in der Landwirtschaft erzeugt auf dem Lande eine immer sërker werdende Schicht von Trakrorenführern, Mähdrescherführern, von Arbeitern der Maschinen- und Trakcorenstationen und der Reparaturwerkstätten für Landmaschinen. Die sozialistischen Arbeitsformen dringen in das Dorf ein und fassen hier Wurzel. Die Kollektivbauernschaft strebt nach Meisterung

Dic Abkürzung des Vornamens von Pašukanis (nJ." beruhe wahrscheinlich aul seinem nucht ins Deutsche überseczeren Vormamen Javgerij. (Rd. KJ). 
der agronomischen Wissenschaft, um sie in den Dienst der sozialistischen Landwirtschaft zu stellen.

Die Arbeiterklasse hac sich selbst in den 18 Jahren der Revolution umgemodelt. Im Okrober eroberte sie die Staarsmacht und die Kommandohöhen der Volkswirtschaft. Sie ist zahlenmäßig gewachsen, hat ihr kulturelles und ideologisch-politisches Niveau gehoben. Sie ist zur fühenden, tonangebenden Schicht der Gesellschaft freier Arbeitsleute von Stadt und Land geworden. Sie formt nach ihrem Ebenbilde die gesamte übrige Masse der Werktärigen. Das sozialpolitische Profil der Angestellten und Intellekruellen hat sich von Grund auf geändert. Es gibc jeczt in der Sowjetunion keine Schicht von Werktätigen, die nicht vom Prozeß des sozialiscischen Aufbaus mil ergriffen wäre, die nicht an sich den Einfluß der proletarischen, kommunistischen Ideologie spüren würde. Die einheisliche sozialistische Volkswirtschaft bildet das solideste Fundament für die politische und ideologische Einheit der national so bunt zusammengesetzten Sowjetunion.

Es gab eine Zeit, wo die Narodniki-Parteien erklärten, ihr Ziel sei, alle werktätigen Klassen zu vereinigen: die Industriearbeiter, die Bauernschaft und die werkràtige Intelligenz. Diese umfassende Autgabe stellcen sie der »engherzigen * Auffassung der Marxiscen-Bolschewiken entgegen, die angeblich nur von den Inceressen des Prolecariats ausgingen.

Lenin und Stalin deckten in ihrer Kritik der Narodniki die ganze Falschheit, die ganze Armseligkeit dieser Versuche von Literaten auf, die Einheit der Werktätigen auf der Grundlage der Erhaltung des Kapitalismus zu postulieren. Erst der Sieg der proletarischen Revolution, erst der Ubergang der Bauernschaft auf die Seite des Proletariats, ersc die sozialistische Umgestaltung der kleinen Warenwirtschaft haben die Bauernsehaft von dem Elend, von der unvermeidlichen Differenzierung, vom Großbavernjoch erlöst. Die Dikiatur des Proletaniats hat ein wirklich einheitliches Volk geschaffen und zusammengefügı, das durch keine Klassengegensätze zerrissen ist.

Dieses Volk, das die uralcen Ketten der gutsherrlichen und kapitalistischen Ausbeutung abgestreift hat, ist jung wie der Staat, den es geschaffen hat. Der Staat der sozialistischen Gesellschaft ist die Kraft, die für die Festigung und den Ausbau der Grundlagen dieser Gesellschaft notwendig ist. Dieser Staat, den das Proletariat anstelle der zerstörten bürgerlichen Staatsmaschine geschaffen hat, hat eine gewaltige historische Rolle gespielt und muß sie noch weiter spielen bis zur höchsten Phase des Kommunismus. Nur Opportunisten, nur Leute, die die Grundlagen der Lehre von Marx und Lenin zu revidieren versuchen, körnen dies bezweifeln. Eine srarke Staatsmacht ist notwendig zur Schaffung der ökonomischen Voraussetzungen der künfrigen staatenlosen, kommunistischen Organisation der Menschbeit. Aufrechterhaltung der staatlichen und betrieblichen Disziplin, Statistik und Kontrolle der Arbeic und des Verbrauchs, Schutz des sozialistischen Eigentums, Bekämpfung der Uberresce von feindlichen Klassenelemencen, Bekämpfung der Uberreste des Kapicalismus im Bewußtsein der Menschen, Verteidigung des sozialistischen Varerlandes gegen Anschläge der Imperialisten - alles dies erfordert einen starken, exakt arbeitenden, hochqualifizierten Staatsapparat.

Der sowjecische Staatsapparat wurde wiederholt umgestellt gemäß den wachsenden Aufgaben des sozialistischen Aufbaus und der Wirtschaft. Die Formen der Sowjerdemokratie haben sich wiederholt geändert, je nach den konkreten Bedingungen des Kampfes um den Sozialismus. Der Aufbau der klassenlosen sozialistischen Gesellschaft leizet eine neue Etappe in der Entfaltung der Sowjetdemokratie ein. Der Ausdrude dieser Etappe ist die Stalinsche Verfassung, die laut Beschlu $\beta$ des VII. Sowjetkongresses ausgearbeitet wird. Die Einführung des allgemeinen und gleichen 
Wahlreches mir geheimer Abstimmung wird es ermöglichen, das Band zwischen der ganzen Bevölkerung und den Staatsorganen noch enger zu knüpfen. Sie wird die Kontrolle der Massen über die Tärígkeic der Staatsorgane verstärken, sie wird dazu beitragen, daß die Arbeiter, Kollektivbauern und alle Werktärigen in noch breiterem Maße zur Verwaltung des Staates und der Organisienung der Produkcion herangezogen werden. Und damit wird der Sowjetstaat im wabren Sinne des Wortes ein Volksstaat.

Eine sowjerstaatliche Angelegenheit ist eine allgemeine Volksangelegenheit. Sie wird verwirklicht im Berrieb und in der Kollekrivwirtschaft, auf dem Treffen der Stachanowarbeiter und in der Tagung der Akademie der Wissenschaften, in der Schule und auf dem Sportplatz. Die Arbeit des Verwaiters, des Gelehrten, des Richters, des Fabrikdirektors, des Häuers, des Stahlgießers, der Kuhmelkerin har in der Sowjerunion in gleicher Weise staatliche Bedeurung. Die sowjerische sozialistsche Demokratie ist auf der Grundlage des sozialistischen gesellschaftlichen Eigentums, ist darauf aufgebaut, daß die Arbeit allgemeine Angelegenteit ist. Die Politik des sozialistischen Staates finder immer mehr ihren Ausdruck in den wirtschafulichen und kulturellen Aufgaben. Technik, Hebung der Arbeitsproduktivitär, Bildungsfragen - damit befassen sich die sowjecischen Kongresse und Konferenzen, damit ist die sowjerische Presse angefüllt, damit befassen sich die sowjetischen Organisationen. Schon 1920, auf dem VIII. Allrussischen Sowjeckongreß sprach Lenin von dem Beginn einer glücklichen Epoche, wo man

won der Politik seltener und niche so lange sprechen wird, wo dagegen die Ingenieure und Agronomen mehr sprechen werdena.

Die Sowjetunion ist jetzt in diese Epoche eingetreten.

Aber wieviel Aufmerksamkeit die Fragen des wirtschaftlichen und kulturellen Aufbaus auch auf sich lenken mögen, die Werkürigen der Sowjezunion können darüber keinen Augenblick die Feinde vergessen, dürfen keine Minute die revolucionäre Wachsamkeit im schonungslosen Kampl gegen die Uberreste der bürgeriichen Konterrevolucion im Lande und gegen ihren Vortrupp, die Hefe des konterrevolutionären Trotzkismus und die Sinowjewleute, gegen die Schädlinge, Spione und Diversanten erschlaffen lassen. Der äußeren Gefahren gedenken, die dem sozialistischen Vaterland von seiten der Imperialisten drohen, die Abwehr des Feindes vorbereiten, die Wehrkraft des sozialiscischen Vaterlandes stärken, den Sowjetpatriotismus fördern - ist erste Pflicht jedes Bürgers der Sowjecunion.

Die Sowjetmacht ist die demokratischste Macht der Welt, und darum ist sie auch eire gefürchtete und drohende Macht für alle Feinde des Sozialismus. Das ganze Land weiß, welch heroischen Dienst die sowjetischen Grenzwächter versehen. Aber der Feind, der Spion, der Diversant hat es nicht nur mit den Grenzwächtern zu tun, sondern auch mit den Koliekrivbauem der Grenzzone, die sorgsam ihren heimatlichen sozialistischen Boden behüren.

Der Sowjecstaat war, ist und wird der Vortrupp der Weltarmee des Soziadismus sein. Ein Schrecken für die Feinde des Proletariats, für die Weißgardisten und die faschistischen Mordbrenner, ist der Sowjetstaas den Werktärigen der ganzen Welt teuer und ans Herz gewachsen. Es gibc kein Land der Welt, wo das Symbol Harnmer und Sichel die Herzen der Arbeiter und Bauern nichr höher schlagen ließe, denn es ist das Zeichen der Hoffnung auf Erlösung vom Joche des Kapitalismus. Die unerschütrerliche Gewißheit, daß das Proletariat die ganze Masse der Werkrätigen mir sich reißen wird, daß es die Produktion und Distribution auf sozialistischer Grundlage zu organisieren verstehen wird, diese Gewißheit durchdringt alle Reden Lenins, angefangen von den ersten Tagen des Bestehens der Sowjetmacht. „Früher schuf der ganze menschliche Geisc «, sagre Lenin auf dem III. Sowjerkon- 
greß, „schuf all sein Genie nur, um den einen alle Wohltaten der Technik und der

Kultur zugute kommen zu lassen, und um die anderen des Aliernorwendigsten, der Aufklärung und der Weiterentwicklung, zu berauben. Jetzt aber werden alle Wunder der Technik, alle Errungenschaften der Kultur allgemeines Volksgut, und von nun an werden der menschliche Geisc, das menschliche Genie niemals auf die Gewaltmittel, auf die Ausbeutungsmittel gerichtet sein. Das wissen wir, und lohnt es sich denn nich, für diese gewalrige historische Autgabe zu arbeiten, alle Kräfte hinzugeben? Und die Werktätigen werden dieses titanenhafte historische Werk vollbringen, denn in ihnen schlummern die gewalcigen Kräfte der Revolution, der Wiedergeburt und Erneuerung.a

So sprach Lenin, als er gegen die kleinbürgerlichen Schleppenträger des Kapitals 1918 auftrac.

- Wir können und müssen die kapicalistischen Elemente unserer Wirtschaft besiegen, wir kónnen und müssen die sozialistische Gesellschaft in unserem Lande aufbauenu, sagte Stalin, als er 1926 gegen die konterrevolutionäre Theorie des Trotzkismus aufrat.

Wie oft wurden diese kühnen Ziele als weltfremde Träume verlacht! Wie oft trompeteten die Feinde, die Bolschewiken wären wklüger geworden«, hätten ihre wweltfremden Träume aufgegeben ", seien wernüchtert«! Und wie oft sind diese Hoffnungen der Lakaien des Kapitalismus zuschanden geworden! Der Bahn Lenins und Stalins folgend, überwand die Arbeiterkłasse ein Hindernis nach dem andern, ohne die angestrengte, mühselige Arbeit zu scheuen, manchmal zurückweichend, um darauf von neuem vorzugehen, dabei jeden Schritt, jede eroberte Position befestigend.

Das, was von den Feinden als *Absage an die Uropie « hingestellt wurde, erwies sich bei Lichte besehen als ein neuer raktischer Zug der Bolschewiki, der ihnen gestattete, rascher und zuversichtlicher vorzugehen.

Das im Oktober 1917 verkündete Programm wurde reale Wirklichkeit. Die politischen und juristischen Möglichkeiten, die von der Dikratur des Proletariats aufgedeckt wurden, haben sich mit konkretem, wirtschaftlichem und sozialem Inhalt angefülls. Die vorgeschrittenste politische Form erbielt ein Fundament in der Gestalt der vorgeschrittensten sozialistischen Okonomik.

Es gibt in der Welt keine andere Staatsmacht, keine andere politische Partei, die auch nur im entfernten Maße sich einer solchen unentwegten Erfüllung eines einmal angenommenen Programms rühmen könnte.

Die Staaten der kapicalistischen Welt und ihre Parteien, in erster Reihe die faschiscischen Staaten und Parteien, die die collwütigste Reakcion verkörpern, sind auf der Lüge, auf dem Berrug der Volksmassen aufgebaut.

Im sowjetischen sozialiscischen Staace schlägt der lebendige Puls der Millionen, die den Weg der Befreiung vom Ausbeuterjoch gefunden haben. Im Sowjetstaate isc die große geschichtliche Wahrheit des siegenden Kommunismus verkörpert.

\section{DIE POLITTSCHE UND WIRTSCHAFTLICHE GRUNDLAGE DER SOWJETUNION [1936]}

Von E. Paschukanis (Moskau)

Die neue Sowjerverfassung, der Entwurf, der dem ganzen Volk zur Beureilung vorgelegt worden isr, stellt ein Dokumenc von weltgeschichtlicher Bedeutung dar. Es ist dies die Charte der Freibeit der Werktätigen, die nicht nur die Herrschaft der Kapitalisten und der Grundgesitzer niedergerungen und auch das politische Joch 
dieser Klassen vernichtet, sondern auch die ganze Wirtschaft des Landes auf die sozialistische Harmonie umgestimmt, die Ausbeuterklassen Liquidien, den Boden selbst, auf dem der Kapitalismus wachsen könnte, vernichter haben.

Während früher, in den Jahren 1918 und $1923 / 1924$, als die erste Verfassung der RSFSR und die Verfassung der Sowjetunion angenommen wurden, die Bezeichnung des Sowjerstaates ais sozialistisch nur die feste Entschlossenheir der Arbeiterklasse zum Ausdruck brachce, bis zu Ende zu kämpfen und den Sozialismus aufzubauen, drücken jetzt die Worte der neuen Verfassung: "Die Sowjetunion ist der sozialistische Staat der Arbeiter und Bauerna (Arrikel I) das aus, was erreichs, erabert und zu einer unabänderlichen Tatsache der Weltgeschichte geworden ist.

Lenins Vermächtnis, das Land der Neuen Wirtschaftspolitik (NEP) zu einem sozialistischen Land zu machen, ist von der Arbeiterklasse und von den Werkrätigen der Sowjerunion unter der Führung des großen Stalin erfüllt worden.

Der Sowjetstaat ist $\nu$ der sozialistische Staat der Arbeiter und Bauerna. Diese Formel der Verfassung festigt den theorecischen und praktischen Sieg der Lehre Lenins und Stalins vom gegenseitigen Verhälınis zwischen der Arbeiterklasse und der Bauernschaft. Die Menschewiki, die Trotzkisten und das übrige gegenrevolutionäre Gesindel haben nicht wenig Bemühungen darauf verwandr, den unversöhnlichen Gegensaiz zwischen den Inceressen der Arbeiter und der Bauern zu „beweisen«, wobei sie bemühr sind, dadurch das Vertrauen der Arbeiterklasse zu ihren eigenen Kräften zu unterwühlen und den Aufbau des Sozialismus zu sprengen. Der Sieg der Kollektivierung war eine vernichtende Antwort der Partei Lenins und Scalins auf diese gegenrevolutionären Erfindungen.

Das Bündnis der Arbeiterklasse und der Bauernschaft hat sich jetzt auf eine neue höhere Stufe erhoben. Die Bauernschait hat unter der Führung der Arbeiterklasse einen enrscheidenden Schritt auf dem Wege zu ihrer Umgestalsung aus kleinen Warenproduzenten zu Mirgestaltern der sozialistischen Gesellschaft gemacht. In der Stadt und auf dem Dorfe herrschen die sozialistischen Produktionsverbältnisse, die kollekrivwircschaftliche Bauernschaft ist unwiderruflich zur roten Fahne des Sozialismus eingerückt. Es geht der Prozeß der Verwischung der Klassengrenzen zwischen der Arbeiterschaft und der kollektivwirtschaftlichen Bauernschaft vor sich. Der Kollekrivwirtschafter wird zum aktiven und bewußren Erbauer der sozialistischen Gesellschaft.

"Die politische Grundlage der UdSSR - besagt Artikel 2 der Verfassung - bilden die Räre (Sowjets) der Deputierten (Abgeordneten) der Werktärigen, die als Resultar der Niederwerfung der Macht der Großgrundbesizzer und Kapizalisten und als Ergebnis der Eroberung der proletarischen Diktacur entstanden und gefestigt sind. . Nur der Weg der Sowjets, nur der Weg der Diktatur des Proletariars und des unentwegten Kampfes der Werkëtigen unter der Führung der Arbeiterklasse und ihrer Vorhut, der Kommunistischen Partei, kann zum Siege des Sozialismus führen. Dies ist die Schlußfolgerung, die die Arbeiter und die Bauern in der ganzen Welt ziehen, wenn sie die neue Sowjetverfassung lesen. Die Sowjermacht ist die echte politische und staatsbürgerliche Freiheir für die Werktärigen, iss die Vernichtung des nationalen Jochs und der schänd̈lichen Nichtgleichberecheigung der Frau, ist die echte breireste Demokrarie.

Die Sowjers der Arbeiter-, Bauern und Rocarmisten-Deputierten erhalten durch die neue Verfassung die Bezeichnung: Sowjers der Depurierten der Werktätigen. Diese Abänderung ist keineswegs zufällig. In ihr drïckt sich die Tatsache aus, daß neben den Arbeitern, Bavern und Rotarmisten im Sowjetland die Angestellien, die Ingenieure, die Techniker, die Pädagogen, die Ärzte, die Gelehrten, kurzum die werktäagen Intellektuelien der Sowjerunion gleichberechtigre Teilnehmer am großen 
sozialistischen Aufbau sind. Diese Anderung drücke den erfolgreichen Prozeß der Ummodlung und der Umerziehung eines beträchtlichen Teiles der alten Inceilektuellen und der Schaffung zahlreicher Kader einer neuen Sorvjetincelligenz aus, die aus den Reihen der Werktätigen, der Kinder von Arbeitern und Kollektivwirtschaftern, die sich durch nichts und in keiner Weise vom werkrätigen Volk loslösen, hervorgegangen ist.

Die Sowjecs der Deputierten der Werktätigen sind die Nachfolger und Fortsetzer jener ruhmreichen Sowjets der Arbeiterdepucierten, die unter der Leirung der Partei der Bolschewiki im Okwber 1917 die Macht übernahmen. Die Erhaluung der Vorhutsrolle der Arbeiterklasse und der Führung der Kommunistischen Partei ist eine notwendige Vorausserzung dafür, daß die Sowjets der Depucierten der Werktäcigen ihre Aufgaben der weiteren Entwicklung und der Fescigung der sozialiscischen Wirschaft, des Schutzes des sozialistischen öffentlichen Eigentums, der Steigerung der Macht der Sowjecunion erfolgreich erfüllen. Die Kommunistische Partei als vorgeschrittene Abteilung der Werktärigen, als wührender Kern aller Organisationen der Werktätigen, sowohl der gesellschaftichen als der staatlichen*, ist die führende Kraft und wird sie bleiben.

Kennzeichnend für die bürgerlichen Verfassungen sind entweder das Verschweigen der wirtschaftlichen Grundlagen des Staates oder aber die Versuche, sich mir allgemeinen, formalen, nichtssagenden Redensarten herauszuwinden.

Schon seit den Zeiten der amerikanischen und der französischen Erklärungen der Menschenrechte am Ende des 18. Jahrhunderts verkünden die bürgerlichen Verfassungsakte das Eigentum als unverbrüchliches Recht des Menschen. Diese dehnbare juriscische Formel sagt über den wirschaftlichen Inhalt des Eigencums einfach nichts. Hinter dieser Formel kann sich sowohl das persönliche Eigentum an Verbrauchsmitteln wie auch das kleine werkzätige Eigencum des Bauern und Handwerkers und auch das Eigentum des Kapiralisten oder des ungeheuerlichen kapitalistischen Trusts verbergen.

Nicht minder verlogen sind auch die späteren Formulierungen, die sich auf die Nachkriegsperiode beziehen. Die Weimarer Verfassung Deurschlands vom Jahre rgrg bemühr sich, den Kapitalismus mir folgenden heuchlerischeo Sätzen zu verschönern: "Der Aufbau des Wirschaftslebens muß den Grundlagen der Gerechrigkeit und dem Ziel der Sicherung eines menschenwürdigen Daseins für alie dienen." (Artikel is 1 . Aus dem Russischen rücküberseczt. Die Red.) Und auf daß es keinen Zweifel daran gäbe, daß sich die Kapitalisten von nun an nicht um den Profit, sondern um den Wohlstand ihrer Arbeiter kümmern werden, fügt Artikel i 53 der gleichen Weimarer Verfassung hinzu: »Eigentum verpflichtet. Seine Nutznießung muß zur gleichen Zeit ein Dienst am allgemeinen Heil sein.u (Aus dem Russischen rückübersetzt. Die Red.)

Diese verlogene und heuchlerische Demagogie berreiben auch die faschistischen Geserzgebungen, indem sie den Kapitalisten als eine Person, die wnationale Funktionen * erfülle, (Arbeitscharte Italiens vom Jahre 1927) und als „Führer im Betrieb* bezeichnen. Das Wesen dieser Redensarten ist das gleiche: Der Arbeiter ist nach wie vor zur Ausbeutung, zur Erwerbslosigkeit und zum Elend verureilt und wird außerdem des Organisacionsrechtes, des Rechts auf Streikkampf gegen den Kapitalistcr beraube.

Die wirtschaftliche Grundlage des Sowjetstaates bilden vas sozialistische Wirtschaftssystem und das sozialistische Eigentum an den Produktionsmitteln. Im gesellschaftichen Außbau der Sowjecunion ist weder für den Großgrundbesitzer noch für den Fabrikanten, weder für den Bankier noch für den Händler oder Spekulanten Platz. In der Sowjecunion ist das kapicaliscische Wirtschaftssystem 
liquidiert, und die Ausbeutung des Menschen durch den Menschen vernichtet. Darin besteht die Grundlage der Grundlagen der Freibeit der Werktätigen, darin besteht ihre Befreiung von der ewigen Drohung der Erwerbslosigkeic, vom Elend und von jeglicher Erniedrigung, von einer Drohung, die in den kapitalistischen Ländern über jedem Arbeiter, über jeden Werkrärigen schwebr.

Das sozialistische Eigentum in der Sowjecunion bestehe nur als staatliches, d. h. dem ganzen Volke gehörendes Eigentum oder als genossenschaftlich-kollektivwirtschaftliches Eigentum. Die Verfassung hebr die gewaltige wirtschaftliche und politische Bedeutung der Tazsache hervor, daß neben folgerichtig sozialistischen Unternehmungen, die sich im Eigentum des Staates befinden, Unternehmungen bestehen, die das gesellschaftliche sozialistische Eigentum der Kollektivwirtschaften und der Genossenschaftsorganisationen bilden. In diesen Artikeln der neuen Verfassung ist das Ergebnis des siegreichen Kampfes um den Leninschen-Stalinschen Weg der sozialistischen Umgestaitung der keeinen Warenwirtschaft befescigt. Das Artel ais Grundform der Produktivgenossenschaften der Sowjetbauernschaft, die die richtigste Paarung der persönlichen und der gesellschaftlichen Interessen sichert, die Vereinigung der Kollektivwirtschaft mir der zusätzlichen Hofwirtschaft des Kollektivwirschafters, die Übergabe des von den Kollekcivwirschaftern besetzten Bodens an sie zu fristloser Nutzung, d. h. auf ewig, alle grundlegenden Bestimmungen der Stalinschen Kollektivwirtschaftssatzung sind als unverbrüchlicher Bestandteil in die neue Sowjetverfassung eingegangen. Sie werden für die viele Millionen umfassende Bauernschaft des ganzen Erdballs als ein Zeugnis dessen erklingen, daß es nur auf der Grundlage des Sowjetaufbaus und der Kollektivwirtschaft möglich ist, sich von der Knechtschaft unter Großgrundbesitzem und Kulaken, von Elend und Ruin freizumachen. Der kollektivwirtschaftliche Aufbau und die Sowjerverfassung sichern jedem Bauern die Hebung des mareriellen und kulturellen Wohlstands auf der Grundlage des kollektiven Besitzes der grundlegenden Produktionsmittel und die sichere, beständige Nutznießung der zusäzzlichen Hofwirtschaft und der zusäzzlichen persönlichen Wirtschaft, während in den kapiralistischen Ländern die Banken und die Wucherer den Bauern mit der Schlinge der Schulden umstricken, ihn zugrunde richren, ihn aus dem Hause jagen, wobei sie ihn zu einem Erwerbslosen, zu einem Obdachlosen machen.

Das sozialistische Wirtschaftssystem bedeutet, daß das ganze Wirtschaftsleben im Sowjetstaat durch einen Volkswirtschaftsplan bescimmt und gelenkt wird. Der gesellschaftliche Aufbau der Sowjezunion kennt weder Oberproduktion noch Krisen noch Erwerbslosigkeit. Der Sowjesstaat, der die Arbeit zu einer allgemeinen Pflicht gemacht hat, sichert jedem Staatsbürger das reale Recht auf Arbeit, das Recht auf Erhalt einer garantierten Arbeit mit Bezahlung der Arbeir entsprechend Menge und Güte.

Wo ist außer der Sowjerunion irgendein Staat imstande, irgend etwas derartiges in seiner Verfassung zu erklären? Die bürgerlichen Regierungen können nicht einmal andeurungsweise der Bevölkenung darlegen, mir welchen Maßnahmen sie auch nur die Erwerbslosigkeit zu verringern beabsichrigen. Und dies ist völlig begreiflich: Kein einziger bürgerlicher Staat ist Herr über die Elementargesetze der kapiralistischen Okonomik. Die Einmischung der Regierungen, die gegen die Krise skämpfen «, läuft auf die systematische Abwälzung der Last der Krise auf die Schultern der Bevölkerung hinaus, indern aus der Stazrskasse den Großkapitalisten Hilfe geleistet wird, und indem die Kriegsindustrie und die Kriegsaufträge gewaltig gesteigerr werden.

Die große proletarische sozialistische Oktoberrevolucion ist die erste Revolution, die nicht nur die Arbeiter und die Bauern vom politischen Joch der Großgrundbe- 
sizzer und der Kapitalisten befreit, sondern ihnen auch eine immer mehr zunebmende Befriedigung der materiellen und kulturellen Bedürfnisse gesichert hat. Die neue Verfassung der Sowjerunion verkündet nicht nur das Recht aut Arbeit, sondern auch das Recht auf Erholung, das Recht auf Sicherstellung im Falle des Airerns, der Krankheit und des Verlustes der Arbeitsfähigkeit, das Recht auf eine Bildung, die sowohl die Grund- wie auch die Mirrel- und Hochschule umfaßt.

Diese Arrikel wie auch die gesamte Verfassung sagen nichts über die Zukunfu über eine ferne Perspektive. In ihnen ist das niedergelegh, was schon jetzt errungen ist, was infolge harnäckigen Kampfes und hartnäckiger Arbeic lebendig geworden ist. Der unentwegte Aufschwung der Planwirtschafr, die stürmischen Tempi der Entwricklung der Produktivkräfte auf sozialiscischer Grundlage, die bereits durchgeführte Verkürzung des Arbeirstages auf 7 Stunden für die überwiegende Mehrheic der Arbeiter und der Angestellien, die alljährlichen Urlaube, das kolossale Anwachsen der Mittel der Sozialversicherung, die Entwicklung der medizinischen Hilfe, die Einführung der allgemeinen Elementarbildung, die Errichtung von Schulen, dies ist die reale Garantie jener Rechre, die kein Staatsbürger irgendeines anderen States außer des sozialistischen hat und haben kann.

Die ersten Äụßerungen der Werktätigen der Sowjetunion zum Entwurł der neuen Sowjerveriassung sind vom Gefühl des Stolzes und des Sowjetpatriotismus erfüllt. Unó den ersten Plarz unter den Außerungen von Arbeitern, Kolleksivwirschaftern und Incellektuellen nehmen Bekundungen der heißen Liebe und der Dankbarkeit für jenen ein, der die Völker der Sowjerunion auf dem Wege des Sieges führte, der der Anreger und Schöpfer der neuen Sowjetverfassung ist, für den größten Mann unserer Epoche: Stalin.

\section{Notizen zu den vorstehenden Aufsätzen von E. Pašukanis}

I.

Bei der Lekcüre dieser Aufsätze von Pašukanis aus den Jahren 1935/Y936 verblüfft schon auf den ersten Blick die offensichtliche Wandlung der Dikrion und Argumentationsweise, die geradezu penetrante negacive Etiketrienng anderer theorecischer (und politischer?) Auffassungen und die formelhafte, durch keinerlei theoretischen wie empirischen Gehalt getrübre, allein durch Bezugnahme auf Lenin, vor allem auf Stalin als richtig und wahr unterstellte eigene Position. Freilich, auch diese Beschreibung der veränderten Diktion von Pašukanis haftec noch zu sehr an der Erinnerung an ihn als Theoretiker: Können ihm die Posicionen, für die er sich in den Aufsätzen scark machc, überhaupt als eigene zugerechnet werden? Ist es noch sinnvoll, von der Etiketrierung anderer Auffassungen' zu sprechen, wenn diese weder in ihren Begründungszusammenhängen dargestellt noch so referiert werden, daß sie wenigstens aus sich heraus verständlich werden? Auch die im schlechten Sinn moralische Art und Weise, mit der Pašukanis die Reihe der cheoretischen Feinde (von den Okonomisten und Menschewiki über die Luxemburgisten und österreichischen Sorialdemokraten, den kleinbürgerlichen Liberalismus und Radikalismus bis hin

1 Die Aulsätze werden im folgenden enzsprechend der Reihenlolge ihres Nachdrucks mit A I, A 2 und A 3 abgekürat, A I S. 40.4 ff. 
zum trotzkistisch-sinowjewistischen Block) ${ }^{2}$ erledigt, indem er sie der "Lüge und Verleumdung des Sowjetstates und unserer Parteik', der noffenen Konterrevolution $\alpha^{4}$ etc. bezichtign, macht deutlich, daß es ihm weniger um Auseinanderserzung (als Uberteugungsversuch durch Argumentation) mit anderen diskutierbaren Ansichten denn um Srigmarisienng mabweichenden theorerischen Verhaltens geht. Diese Schwierigkeic, die angeblich «eigene Meinung dem Auror, die angeblich "fremde Meinung « den Kritisierten zuzurechnen, legt die Vermunung nahe, daß das integrierende Zentrum dieser Aufsäize von Pašukanis nicht das sIch « des reflekrierenden Theoretikers Pašukanis ist, sondern außerhalb und jenseits seiner individuellen Person gesucht werden muß. Damir entfällt aber auch die Möglichkeit, diese Studien als theorecische und wissenschaftliche, nämlich durch die Erkenntnisleistungen des reflektierenden Subjekts vermittelte, zu diskutieren. Pašukanis hätte wohl auch selbst einen soichen Anspruch, mit diesen Aufsätzen als theoretisch reflektierendes Subjekt zu publizieren, weic von sich gewiesen: eine derartige Rolle kann von keinem Individuum ohne Verstoß gegen die Bestimmung der "Diktatur des Proletariass , wie sie Stalin formuliert hat und Pašukanis affirmativ aufnimmt, eingenommen werden. Diese besteht danach nämlich sin leitenden Weisungen der Partei, plus der Durchführung dieser Weisungen durch die Massenorganisationen des Proletariars, plus ihrer Verwirklichung durch die Bevölkenung 's - für individuelle, d. h. der Möglichkeit nach auch immer abweichende Einsichten und entsprechende pragmatische Handlungsalternaciven ist hier per definitionem kein Platz, wäre Verrat an der bestehenden Herrschaftsordnung.

Integrierendes Zentrum dieser Aufsäıze sind daher eirzig und allein die $\rightsquigarrow$ Weisungen der Partei . Indem aber nicht mehr kommunizierende Subjekre, sondern eine gegenüber der Gesellschaft verselbständigte Herrschaftsinstitution darüber entscheider, welche Einsichten und Aussagen intersubjekriv anzuerkennen und daher verbindlich sind, verliert die individuell publizierte Meinung ihren Charakter als Teilnahme am wissenschaftlichen Diskurs: Sie wird zur Propaganda, zur Werbung von Vertrauen in die Aussagen der Partei. Die hier wieder abgedruckten Aufsätze von Pašukanis sind somit Zeugnis eines intellekcuellen Stalinisierungsprozesses, dessen Kurzfassung die keineswegs ironisch gemeint Formel ist: „Die Partei hat immer recht".

II.

Mit dieser Charakterisienung der Autsätze von Pašukanis aus dem Jahr 1935 steht freilich auch der Nuczen in Frage, der ihre emeute Publikation machen soll: Der Informacionswert von Propaganda iss bekannclich gering, jedenfalls in bezug auf den Gegenstand, von dem die Propaganda zu reden meint. Die Sprache, gereinigt von jeder Differenzerfahrung von Realitäc und Ideologie, wird zur parherischen Beschwörungsformel: "Das Land, das keine Krisen kennt, das Land, wo es keine Arbeicslosigkeit gibr, das Land, das mic der nationalen Unterdrückung aufgeräum hat, das Land, das die völlige Gleichberecheigung der Frau gewährleistec hac, das Land, wo jeder Werktätige des morgigen Tags gewiß ist und weiß, daß dem weiteren Aufschwung des öffentlichen und privaten Wohlstandes keine Grenzen geseczt sind, 
dieses Land ist die Sowjetunion, der Staat der freien Arbeitsleute in Stadt und

Land $\alpha^{6}{ }^{6}$

Relevant wird die Beschäftigung mit Propaganda erst dann, wenn nach ihrer Funkrion gefragt wird. Diese Frage kann in zweierlei Richtung gehen: Einerseits wäre zu untersuchen, auf Grund welcher Sirukturmerkmale oder Problemlagen ein politisches System die Institution der Propaganda an die Stelle kritischer (Sozial-) Wissenschaft setzc. Andererseits kann die Funktion von Propaganda daraufhin untersucht werden, zu welchem Zweck sie jeweils konkret eingesetzt wird, welches gesellschaftliche Problem jeweils mit den Mitreln der Propaganda win Szene gesetzt * wird.

Die Einebnung öffendicher politisch-theoretischer Kontroversen auf platte Propaganda geht zwangsläufig einher mit einem erheblichen Realitätsverlust des politischen Syscems in bezug auf innere Spannungen und Widersprüche. Deuclich wird dieser Realitätsverlust an den Inhalten der Propaganda selbst: So wird Pašukanis nicht müde zu betonen, daß Krise und Arbeitslosigkeit beseitigt seien, daß „die Diktatur des Proletariats ... ein wirklich einheitliches Volk geschaffen und zusammengefügt (hat), das durch keine Klassenunterschiede zerrissen ist " 7 , daß ${ }^{2}$ die Sowjetmacht ... die demokratischste Macht der Welt (ist) ${ }^{8}$. Diese Propagandaformeln negieren soziale Krisen, Gegensätze und Konflikte, harmonisieren das soziale Gesamtsystem, betreiben - wie alle Propaganda - explizite und inceressierte Realitätsverleugnung. Solche Realitätsverleugnung fordert allerdings ibre Kosten: Da die bestehenden, aber nicht chematisierten, sondern pauschal geleugneten sozialen Probleme im Interesse der Aufrechterhaltung und Sichenung des etablierten Herrschafissystems gleichwohl wbearbeicet * werden müssen, muß zwangsläufig auch dieser Prozeß der nBearbeitung « sozjaler Probleme und Kontlikre ebenso dementiert werden wie diese selbst. Damit wird mit dem Bereich öffentlicher politischtheorerischer Auseinanderserzung zugleich der gesamte Sektor staarlicher Policik nicht-öffentlich, staacliche Verwaltung agiert - jenseits propagandistischer Verlautbarungen - unter Ausschluß der Offenclichkeit.

Diese Konsequenz der Negation sozialer Konflikre, die Tabuisierung der Diskussion scaatlicher Politik, wird in den Aulsätzen ebenfalls sehr deutlich. Drei unterschiedliche Strategien lassen sich ausmachen, mit denen dieses Tabu aufgebaut wird. Zunächst konstruiert Pasukanis eine vorgebliche Identität von Volk und Staat, womit jede Angelegenheit und Tärigkeit zu einer staatlichen um- und aufgewertet wird, daher sraacliches Handeln jegliche Spezifik verliert, die auch nur entfernt an einen organisierten Herrschaftszusammenhang erinnern könnte: „Eine sowjetstaatliche Angelegenheit ist eine allgemeine Volksangelegenheit. Sie wird verwirklicht im Betrieb und in der Kollektivwirtschaft, auf dem Treffen der Stachanowarbeiter und in der Tagung der Akademie der Wissenschaften, in der Schule und auf dem Sportplatz. Die Árbeit des Verwalters, des Gelehrten, des Richters, des Fabrikdirekcors, des Häuers, des Stahlgießers, der Kuhmelkerin hat in der Sowjecunion staatliche Bedeutung ${ }^{9}$. Diese Universalisierung des Bereichs staatlicher Angelegenheiten wird sodann ergänzt durch eine Reduktion des Politikbegriffs auf Maßnahmen zur Erhöhung der Arbeitsproduktivitär: „Die Politik des sozialiscischen Staates findet immer mehr ihren Ausdruck in den wirtschafulichen und kulturelien Aufgaben. Technik, Hebung der Arbeitsproduktivitär, Bildungsfragen - damit befassen sich die sowjetischen Kongresse und Konferenzen, damit isc die sowjetische Presse

$6 \mathrm{~A}=2$ S. 408

7 A 2 S. 409 .

8 A 2 S. 410

$\rightarrow$ A 25.410 . 
angefülle, damil befassen sich die sowjetischen Organisationen ${ }^{10}$. Dem entpolitisierten Politikverständnis entsprechend findet eine öffentliche Debatte nur mehr statt über Okonomie und Effizienz des produktiven Einsatzes von Menschen und Maschinen (und nur hier, daraut verweisen die Unterstreichungen im Text, darf sie stattfinden). An die Stelle der Diskussion alternativer Politiken, alternativer Organisationsweisen der Produktion und der Distribution, anderer Formen des Lebens und der Kommunikation trit die Diskussion über Technologien. Staatliche Herrschaft, Politik wird zur Technik entpolitisiert, das Ende einer Ära scheint gekommen, in der die Hoffnungen auf ein gutes und humanes Leben sich an die Politik richreten: "Schon 1920, auf dem VIII. Allrussischen Sowjetkongreß sprach Lenin von dem Beginn einer glücklichen Epoche, wo man svon der Politik selcener und nicht so lange sprechen wird, wo dagegen die Ingenieure und Agronomen mehr sprechen werdenc. Die Sowjetunion ist jetzt in diese Epoche eingetreten ${ }^{11}$. Die dritte Strategie, mit der Pašukanis das Tabu öffentlicher Diskussion über Politik absicher, ist die Stigmatisierung jedes öffentlichen Diskussionsansatzes, welcher sich den herrschenden Propagandaformeln verweigert, zum hochverräterischen Anschlag auf die "Sowjetmachr«, zur "konterrevolutionären Theorie.

Die harmonisierende Propaganda der aufgelösten sozialen Widersprüche schlägt bruchlos um in unversöhnliche Feindbilder, ja in offene Denunziation. Damit wird in diesen Aufsätzen von Pašukanis selbst noch der Mechanismus erkennbar, der an die Stelle öffentlicher Auseinandersetzung über staatliche Politik und soziale Krisen und Konflikte tritr: An die Stelle rationaler Diskussion und Interessenarrikulation tritt nicht die Inscitution der Propaganda allein, sie wird begleitet von ihrem Schazten, dem Terror. Pašukanis ist diesem mörderischen Mechanismus wenig später selbst zum Opfer gefallen, hingerichtet von den Schergen Stalins. Der Irracionalität eines polizischen Sysrems, welches den Realicätsverlust, der mit der Ausschaltung öffenticher politischer Auseinandersetzung zwangsläufig einhergeht, durch die Mechanismen der Propaganda und des Terrors zu kompensieren sucba, kann mit den individuell durchaus rational scheinenden Strategien der Anpassung und Apologie jedenfalls dann nicht entronnen werden, wenn die sozialen Ursachen der terroristischen Systemveränderung nicht begriffen sind ${ }^{12}$ - dies lehrt die Tragik des Intellekruelenschicksals von Pašukanis unrer der stalinistischen Herrschaft. Der - soweit bekannc - leczie Satz, den ex überhaupt publiziert hat und mit dem er den hier wiederveröflentlichten Aufsatz "Die politische und wirtschafuliche Grundlage der Sowjecunion « bescbließt, liest sich heute wie die absurde sacirische Todesmelodie, mit des das Opfer seinen Henker preist: "Und den ersten Plarz unter den Außerungen von Arbeitern, Kollektivwirtschaftern und Incellekruellen nehmen Bekundungen der heißen Liebe und der Dankbarkeit für jenen ein, der die Völker der Sowjetunion auf dem Wege des Sieges führte, der der Anreger und Schöpfer der neuen Sowjetverfassung ist, für den größten Mann unserer Epoche: Stalin. a"s

III.

Stand im Rahmen dieser Ausführungen bisher - soweit sich dies aus den Aufsätzen von Pašukanis direke erschließ - die Funktion und die Wirkung von Propaganda

$10 A_{2}$ S. 410, Hervorhebungen daselbst.

If $A$ iS. 410 .

$12 \mathrm{~V}_{\mathrm{g}}$, hierzu dic Aufsäzzc von Josel Brink und Richard Lorenz, in diesem Hefi S. 341 II. und S. 364 If. jeweils mir weiteren Nachwersen.

1) A 3 S. 45 . 
für das gesellschafcliche Syszem, welches sich ihrer als Institution bedient, im Vordergrund, so soll nun jener anderen Frage nach der Funktion der Propaganda nachgegangen werden: Zu welchem Zweck sie je konkret eingesetzr wird, weiches gesellschaftliche Problem mit den Mitreln der Propaganda inszeniert, agelöst a wird. Die Beantwortung dieser Frage wirft erhebliche Schwierigkeiten auf. Angesichts des minimalen Informations- und Reflexionsgehaltes sind propagandistische Schriftstücke keineswegs aus sich selbst heraus dem Leser zugänglich und verständlich. Gewiß kann man aus dem apellativen Charakrer einer Reihe von stereotyp wiederkehrenden Formulierungen auf eine generelle Zielrichrung der propagandiscischen Absichten schließen. Deutlich wird dies in den Arbeicen von Pašukanis einmal in Richrung auf die Anerkennung und Durchserzung der unhincerfragbaren Ausorität der Partei gegenüber allen anderen gesellschaftlichen und politischen Institutionen: * Die vom VII. Sowjeckongreß beschlossenen Änderungen in der Sowjetverfassung schaffen die Voraussetzungen für ein noch stärkeres Wachstum der Autorirät und des Einflusses der Partei, für eine noch größere politische Akrivitär der Arbeiter und Kollekuvbauern-Massen im Kampfe um die Vernichtung der Uberreste des Kapitalismus, für die Basis unserer Gesellschaftsordnung: das sozialistische, gesellschaftliche Eigentum ${ }^{r}{ }^{\prime 4}$, Die Kommunistische Partei als vorgeschrittene Abreilung der Werktärigen, als sührender Kern aller Organisationen der Werktätigen, sowohl der gesellschafilichen als der staaclichenc, ist die führende Kraft und wird sie bleiben w. 's Ferner propagieren diese Schriften die Norwendigkeit eines *starken Siaates *: $n$ Eine starke Staatsmacht ist notwendig zur Schaffung der ökonomischen Voraussetzungen der künftigen staatenlosen, kommunistischen Organisation der Menschheit. Aufrechterhaltung der staatlichen und betrieblichen Disziplin, Statiszik und Kontrolle der Arbeit und des Verbrauchs, Schutz des sozialistischen Eigentums, Bekämpfung der Uberreste von feindlichen Klassenelementen, Bekämphung der Uberreste des Kapitalismus im Bewußtsein der Menschen, Verteidigung des sozialiscischen Vaterlandes gegen Anschläge der Imperialisten - alles dies erfordert einen starken, exakt arbeitenden, hochqualifizierten Staatsapparat $\alpha .{ }^{16}$

Zwei weicere Aspekte der politischen Encwicklung der Sowjetunion werden von Pašukanis derart häufig angesprochen und dies mit dem schon bezeichneten, beschwörenden Gestus, der sich schon der Form nach gegen jeden Widerspruch inmunisiert, daß sich die Vermutung einer konkreten Realicäts- und Problemverleugnung vermittels dieser propagandiscischen Beteuerungen geradezu aufdrängt. Zum einen ist dies die Formel, mit der er seinen Aufsatz "Volksstaat Sowjetunion* einleiter und die auch in seiner Kommentienung der staljniscischen Verfassung von 1936 breiten Raum einnimmt: „Die sozialistische Gesellschaft der Werktätigen ist in der Sowjetunion bereits aufgebaur ${ }^{17}$, "Die Idee des Sozialismus ist in die Tat umgesetzt $\kappa^{18}$, Im sowjetischen sozialistischen Staate schlägt der lebendige Puls der Milionen, die den Weg der Befreiung vom Ausbeuterjoch gefunden haben. Im Sowjetstaate ist die große geschichtliche $\mathbb{W}$ ahrheit des siegenden Kommunismus verkörperz"'. Ganz deutlich stellt Pašukanis diese behauptete historisch neue Qualität, die mit der Formel wsozialistischer Staat a bezeichner wird, in der folgenden Passage heraus: "Wahrend früher, in den Jahren 1918 und $1923 / 24$, als die erste Verfassung der RSFSR und die Verfassung der Sowjetunion angenommen aurden, 
die Bezeichnung des Sowjerstaares als sozialiscisch nur die feste Entschlossenheir der Arbeiterklasse zum Ausdruck brachte, bis zu Ende zu kämpfen und den Sozialismus aufzubauen, drücken jetzr die Worte der neuen Verfassung: >Die Sowjerunion ist der sozialisrische Sraar der Arbeicer und Bavern' (Arrikel I) das aus, was erreiche, erober und zu ciner unabänderlichen Tatsache der Weltgeschichte geworden ist $\alpha^{20}$. Damit sei, so fährt er fort, "Lenins Vermächtnis, das Land ... zu einern sozialistischen Land zu machen ... unter der Führung des großen Stalin erfüllt worden ${ }^{21}$. Die Verfassung dekreriert und die Propaganda versichert, daß der Sozialismus als Ubergangsstadium zum Kommunismus in der Sowjeturion - wie die noch heute gebräuchliche Formel besagt - preal existierı , zu einer jeder kritischen Fragestellung, jedem Zweifel und jeder geschichrlichen Alternarive enthobenen sunabänderlichen Tatsache« geworden ist.

In engem Zusammenhang mit diesern Topos der erreichten Srufe des «sozialistischen Staatesa stehe der andere, ebenfalls ein offenbar zentrales gesellschaftliches Problem in eine quasi ontologische Aussage verkehrende Topos einer neuen Qualität des Bündnisses der Arbeiterklasse mit der Bauernschaft: "Das Bündnis der Arbeiterklasse und der Bauernschaft ist in eine neue Phase eingetreten, denn sein Hauptziel - die Umwandlung der gestrigen kleinen Warenproduzenten in akcive Erbauer der sozialistischen Gesellschaft - ist erreicht $\alpha .{ }^{22}{ }^{2}$ Das Bündnis der Arbeirerklasse und der Bauernscbaft - so formuliert Pašukanis fast glejchlautend in seinem Aufsarz zur neuen Verfassung - whar sich jetze auf eine neue höhere Srufe erhoben. Die Bauernschaft hat unter der Führung der Arbeiterklasse einen entscheidenden Schritt auf dem Wege zu ihrer Umgestaltung aus kleinen Warenproduzenten zu Mirgestaltern der sozialistischen Gesellschaft gemacht. In der Stadt und auf dem Dorfe berrschen die sozialistischen Produktionsverhältnisse, die kollektivwiruschaftliche Bauernschaft ist unwiderruflich zur roten Fahne des Sozialismus eingerückt. Es geht der Prozeß der Verwischung der Klassengrenzen zwischen der Arbeiterschaft und der kollekcivwirtschaftlichen Bauernschaft vor sich. ${ }^{2}$ Neben der an den Schöpfungsmychos des Alten Testaments angelehnten Sprachhülse, daß die Arbeiterklasse "nach ihrem Ebenbilde die gesamte übrige Masse der Werktätigen (formt) $\alpha^{24}$, wird auf zwei Faktoren verwiesen, die diesen Prozeß der "Verwischung der Klassengrenzen * bedingen: Die Zwangskollekcivienung der Landwirsschaft, als -Sieg des gesellschaftlichen sozialistischen Eigentums "as bezeichner, und die Technisierung der landwirtschaftlichen Produkcion, durch die wsozialisrische Arbeitsformen in das Dorf ein(dringen) $\alpha^{26}$.

IV.

Zusammenfassend lassen sich den Aufsärzen von Pašukanis vier zentrale Propagandathemen entnehmen: Die höhere Stufe des Bündnisses der Arbeiterklasse und der Bauernschaft, die höhere Stufe des nunmehr sreal exisrierenden" sozialistischen Staates, die Norwendigkeit eines starken Stzatsapparats und die Stärkung der Autorität und des Einflusses der Partei. Diese im Wege der Textexegese gewonne-

$20 \mathrm{~A}_{3} \mathrm{~S} .412$. Hervorhebungen daselbst.

2) A S. 412 .

$22 \mathrm{~A}=\mathrm{S} .408$.

2) A S. 412 .

24 A $\ S .409$.

is A 2 S. 408 .

26 A 1 S. 408. 
nen wesenclichen Topoi der Aufsärze verweisen auf mutmaßliche gesellschafcliche wie politische Problemlagen, die mit der Kollektivierung der Land́wirtschaft, der Ausrottung der Kulaken, der Zentralisierung des Staatsapparats und der Beseitigung der innerparteilichen Opposition entstanden oder zumindest verstärkt worden sind. Freilich erlaube die verschlüsselte und formelhafte Sprache, mit der diese Problemlagen propagandistisch inszeniert werden, nicht, die Beziehung zwischen diesen unterschiedlichen Problemzonen näher zu charakterisieren; ebensowenig kann die spezifische Ausprägung der einzelnen Xonfliktbereiche der Propaganda selbst entnommen werden. Dem heutigen Leser dieser Schriften bleibt daher verborgen, ob sie stärker politisch-strategische Interessen (an forcierter Zentralisierung und Erweiterung der Machtbefugnisse von Parteiführung und Staat) oder legitimatorische Absichten (zur Begründung der Noctwendigkeit einer bereits abgeschlossenen Entwicklung) verfolgen, ja ob sie solche Interessen und Absichten überhaupt verfolgen und nicht nur äußerlich - dem herrschenden Sprachgebrauch angepaßt - diese zu verfolgen vorgeben, aber »zwischen den Zeilen « für den zeitgenössischen Adressaten erkennbar eher gegenteilige Signale ausstrahlen. Damit ist die Hauprschwierigkeir benannt, der der Interpretationsversuch propagandistischer Schriften begegnet: Weil ihr wintegrierendes Zentrum " in den gestanzten Formeln der herrschenden Sprachdiktatur zu suchen ist, wird die Entschlüsselung der oktroyierten Skiavensprache zum schwer zugänglichen Spezialgebier einer hiscorisch-politischen Detailwissenschaft (durchaus vergleichbar der modernen geheimdienstlichen "KremlAstrologie ). Zu ihrem adäquaten Verständnis genügt es nicht allein, sie in ihrem historischen, sozialgeschichtlich-politischen Kontext zu interpretieren; vielmehr wird ihr spezifischer Aussagegehalt im Hinblick auf besondere individuelle Konnotationen des Autors erst sichtbar, wenn danüberhinaus das gesamte System der offizjellen - wie der gerade noch zugelassenen offiziösen - policischen ncodes $\alpha$ in die Analyse mit einbezogen wird.

Ohne detaillierte Kenntnis des feingesponnenen ngrammatikalischen Netzwerkes* der jeweils bestehenden herrschaftlichen Sprachregelungen ist es daher gänzlich unmöglich, Schriften wie die hier wiederveröffentlichten Aufsäzze von Pašukanis daraufhin zu uncersuchen, ob sie sich in platter Apologie erschöpien oder hie und da versteckte Kritik anmelden. So mögen Formulierungen wie die, daß ndie Einführung des allgemeinen und gleichen Wahlrechts mit geheimer Abstimmung ... die Kontrolle der Massen über die Tätigkeit der Staatsorgane verstärken (wird) « ${ }^{26 a}$, indem diese Formulierung implizit die Norwendigkeit einer verstärksen dernokracischen Kontrolle der Staarstätigkeit voraussetzt, als politischer Dissens gegenüber der herrschenden politischen Encwicklungstendenz zu deuten sein, die er an anderer Sielle als "Konzentrierung aller Mirtel des $Z$ wangs und der Uberzeugung zum Schutze, zur Festigung und Entwicklung des sozialistischen Eigentums " ${ }^{27}$ charakterisiert. Indes wäre eine solche kritische Position noch vergleichsweise offen formuLert. Ebensogut kann bereits die besondere Auswahl von Zitaten, die scheinbar nebensächliche Reihenfolge rituell standardisierter Beteuerungsformeln, die Abfolge von Argumentationsketten oder das Erwähnen bescimmer Namen und Posirionen - sofern von den offiziellen Propagandastandards abweichend - als politische Opposition erkennbar werden. Wie wernig eine solche UUberlegung eine übermäßige Pointierung der Funktionsveränderung von Sprache unter Bedingungen von Zensur und Propaganda darstellt, zeigt das Phänomen, daß nicht nur die in solchen Schriften enchalkenen Aussagen, sondern mindescens ebenso das Weglassen bestimmter Formeln, Nichterwähnen von Personen zum Element der authentischen 
Interpretation wird. Bei aller durch und durch propagandistischen Form dieser Aufsäzze von Pašukanis wird daher die Frage offen bleiben müssen (jedenfalls im Rahmen dieser Arbeit), ob er als Apologet oder Dissident dem staliniscischen Terrorregime zum Opfer fiel. ${ }^{272}$

V.

Diese hier aufgezeigten Mechanismen sind nicht allein auf politische Systeme beschränkt, die wir als Diktaturen bezeichnen; in Ansätzen lassen sie sich auch bei uns aufweisen: Gesinnungsüberprüfung und formelhafter Bekenntniszwang im Kontext der Berufsuerbotspraxis, Distanzierungsptlicht von der Praxis radikaler Systemopposition, Distanzierungspflicht aber auch von der Sprache, den Denkmustern und Reflexionen solcher Individuen oder Gruppen, für die stellvertretend der Götringer 'Mescalero genannt sei. Im Kern verfolgen derarrige Tendenzen die gleichen Funkwionen, die wir bei der Analyse der Texte von Pašukanis aufgewiesen haben: Die Dethematisierung staatlicher Politik (und von Alternariven zu ihr), die Eingrenzung (und tendenziell die Ersetzung) von kontroverser öffentlicher, insbesondere sozial- und politikwissenschaftlicher Diskussion, die Mobilisierung von Konsens und Loyalieät durch das Gegenstück von Aufklärung: durch die, wie Pašukanis schreibt, "Konzentrierung aller Mittel des Zwangesu. So sehr die akruelle policische Verfassung der Bundesrepublik auch enterne ist von der Entfairung und Totalisierung solcher Mechanismen wie uncer dem stalinistischen Regime, so beunruhigend muß indes auch nur jeder Schritt in eine derartige Richtung sein, die hinter die erreiehte Rationalizät von Herrschaft und staaticher Politik der bürgerlichen Gesellschaft zurückfällt und Herrschaftstechniken und -formen reetabliert, die mit dem Feudalismus übermunden schienen: Die Rirualisienung von Offentlichkeit, Politik und Staat zu einer einzigen gigantischen zeremoniellen Veranstaltung, zum Kult des (meist in "Führern personalisierten) Gewaitmonopolisten "Staas", die Degradierung des Menschen zum willenlosen Arbeitscier, zum Objek staarlicher Naturbeherrschung und ihrer techniziscisch verkürzten Produkrivitätslogik, die Enteignung aller Freiheiten, selbst noch der Rede- und Denkfreiheit durch sprachliche Verkehrsregelungen, die Greie Assoziacion unter Zensur und Strafe stellen zugunsten einer rigiden Hierarchie gestanzter Symbole. Wobei allerdings solch moderner "Feudalität * jegliches Element fehlt, welches Marx noch von ngemütlichen Knechtschaftsverhältnissen in bezug auf die historisch vergangene Epoche zu sprechen erlaubce: Die Toralizät des staatlichen Zugriffs auf das Individuum kennt

$27 a$ Diese Frage ist, soweir ich sehe, in der einschlägigen Lireraur bisher nichr eingehender untersucht wordep. Daß das Bild von Pašukasis als überzeugern Anhänger des Stalinismus, welches die vorstehend wieder publizienen Aulsäue auf den ersen Blick verminteln, vermudich unzutroffend is, kann einigen Passagen aus der Untersuchung von L. Schapiro entnommen werden. So qualifizier er den ersten, bereits erwähnten Widermul von Pasukanis aus dern Jahr r9jo als "zweideuvig*, weil cr wauch als solcb versteckte Knrik im Niedergang der Gesctzlichkeit im sowjerischen Sctat erschuenco sein (mag)×a. a. O. S. 495 und berichtet, da $B$ sich scine späuce offizielle Verfemung und Ermordung auch daraus erklörn, daß er den stalinistischen Terrorprakriken rechtliche Schranken scezen wollre: : Tausächlich kann niche bezwcifelt werden, daß einer der Gründe, die Pašukanis' Sehicksal zu einer Zeit besiegclten, als Stalin aul seine Linie des uneingeschränkten Terrors einsehwenke, diuin zu sehen ist, daß er der Verlasser des Enrwarfs eines neucn Sirafyeserzbuches lür die UdSSR war. Diescr Enrwur schlaß, unter anderen Bestimmungen, offenbar die Todesstrafe aus und sab in den Zwangsarbeislagem cin stark gernilderes Regime vor. Obwohl dieser Entwurt Pašsteanis' nicht veröflentlicht worden war, hieß es doch, daß er innerbalb der Abreilung Recht der Kommuniscischen Akademie, deren Vorsitzender Pasukanis war, große Wirkung gehabr habe. Daß Vyšnskij nach dem Srurz Pašukanis' dessen Posten einnahm, machte diesen sliberalen. Tendenzen bald ein Ende, und des Strafgcsevzbueb solle fur weitere zweiundzwanzig Jahere nichr mehr revidicr werden $x_{2}$ 2. A. O. S. 433 f. 
keine Brechung mehr durch die bunte Vielfalt partikularer und konkurrenter Gewalten, das zum vereinzelten Einzelnen monadisierte Individuum genießt weder patrimonialen Schutz und Fürsorge noch die Gewißheit der selbstverständ́lichen Unterstützung durch die ständisch-familiale Gemeinschaft. Die Zerschlagung jeglicher Partikularitäten, die dem mit dem Gewaltmonopol Staat identifizierten Monopolanspruch der Partei entgegenstehen könnten, ist denn auch durchgängiges Motiv dieser Texte von Pašukanis: Der Weg zum Kommunismus wird dargestellt als sozialer Prozeß der Uniformierung, in dem alle gesellschaftlichen Verhältnisse wie auch die Menschen selbst auf einen identischen Sachverhalt, einen einheirlichen Begriff reduziert werden, die Basis unserer Gesellschafrsorónung: das sozialistische, gesellschaftliche Eigentum $\alpha^{28}$, "die sozialistischen Arbeitsformen $\alpha^{29}$ und die Arbeiterklasse, die "nach ihrem Ebenbilde die gesamte übrige Masse der Werkrätigen (formt) ${ }^{30}$.

Voraussetzung dieses Uniformierungsprozesses ist die diktatorische Entesselung der staaclichen Gewalt, ihr Resultat die Produktion eines neuen, einheitlichen Menschentypus, dessen wesentliche Bestimmung in seiner Arbeit liegt: Der Werktäcige. Die Analyse des scalinistischen Terrors hat als eine seiner zencralen Funkrionen genau dies herausgearbeiter, die Liquidierung der revolucionären Schicht der Intelligenz, die nach der Okroberrevolution den Typus des Funktionärs in Wirtschaft, Partei, Militär und Staat bestimmt hatte, deren Platz nun ein neuer, zwangsiveise entpolitisierter Funktionärstypus einnimmt: der technologische Kader. " Pašukanis, dafür sprichr die nachfolgende Passage aus seiner Kommentienung der neuen sowjetischen Verfassung, hat diese Funktion durchaus erkannt: Die Sowjets der Arbeiter-, Bauern und Rotarmisten-Deputierten erhalten durch die neue Verfassung die Bezeichnung: Sowjers der Depurierten der Werktätigen. Diese Abänderung ist keineswegs zufällig. In ihr drücke sich die Tatsache aus, daß neben den Arbeitern, Bauern und Rotarmisten im Sowjetland die Angestellten, die Ingenieure, die Techniker, die Pädagogen, die Ärzte, die Gelehrten, kurzum die werktätigen Incellektuellen der Sowjerunion gleichberechrigte Teilnehmer am großen sozialiscischen Aufbau sind. Diese Änderung drückt den erfolgreichen Prozeß der Ummodkung und der Umerziehung eines beträchtichen Teiles der alten Intellektuellen und der Schaffung zahlreicher Kader einer neuen Sowjecintelligenz aus, die aus den Reihen der Werkrätigen, der Kinder von Arbeitern und Kollektivwirtschaftern, die sich durch niches und in keiner Weise vom werkrätigen Volk loslösen, hervorgegangen ist ${ }^{32}$. »Ummodlung und Umerziehung der alcen Intellekruellen a sind die euphemisuschen Vokabeln für den Prozeß der Gleichschaltung und Vernichtung der sich noch als politische Akteure - und nicht als technologischer Sachwalter - verstehenden Intellekruellenschicht. Dieser objektiven Systemlogik des stalinschen Sozialismusmodells, der beispiellosen Regression eines sozialen Emanzipationsversuchs, in deren Verlauf die neue Gesellschaft ihre "Eltern « frißr, dieser Syscemlogik war durch individuelle Definitionsversuche nach dem Muster demonstraciver Selbstgleichschaltung - dies lehren neben Pašukanis zahllose Inrellekcuellenschicksale - nicht zu entrinnen.

Wobei das historische Novum der stalinistischen Regressionslogik darin liegt, daß angesichts der fast durchgängigen Selbstanklagen der vors Tribunal gestellten Intellekruellen in den Moskaver Schauprozessen sogar daran gezweifelt werden muß, ob 
derartige zur Schau gestellte Formen der Selbstgleichschaltung überhaupt von dem scheinbar so selbstverständlichen Bestreben getragen waren, der terroristischen Systemlogik zu entrinnen. Dieser Zweifel impliziert die geradezu gespenstische Perspekrive, daß die Äußerungen der Angeklagren in den Schauprozessen subjektiv von der Uberzeugung getragen sein könnten, daß der historische Prozeß̊ der Errichtung des Sozialismus mit Norwendigkeit die Vernichrung ihrer individuellen Existenz fordere; daß sie aus der Einsicht in diese Notwendigkeit das Opfer ihres Lebens "freivillig « bringen aürden, um der Emanzjpationsgeschichte der Gatcung nicht länger im Wege zu stehen. Schon die bloße Möglichkeir einer derartigen, gesellschaftlich produzierten Perversion elementarer, in der Triebstruktur der Individuen verankerter vitaler Lebensbedürfnisse in einen eine ganze soziale Schicht erfassenden Todestrieb, der sich nicht lediglich in den Dienst ejner Sache stellt, die das individuelle Leben kosten kann, sondern in der die systematische Liquidierung der ursprünglicben Revolutionäre sich als der Fortschricz der Sache selbst darstellt, verweist auf eine Struktur des politischen und sozialen Systerns, die die Dimensionen unseres Vorstellungs- und theorerischen Erklärungsvermögens sprengt. Damit muß die Frage nicht nur unbeantwortet bleiben, ob diese vermutlich letzren Schriften von Pašukanis als die eines Apologeten oder Dissidenten des Stalinismus anzusehen sind; schon diese Frage kann sich als faisch gestellt erweisen mit ihren zwei Alrernativen, die die dritte ausschließen: Daß er von der Norwendigkeit dieser Entwicklung durch und durch überzeugt war - auch wenn dieser Weg nur über seine Leiche fortgesetzt werden konnte.

VI.

Auf Grund dieser Uberlegungen erweist sich auch die Suche nach einer erwaigen Kontinuität bzw. Diskontinuizät der rechtstheorerischen Positionen in den Publikationen von Pašukanis als ein ausgesprachen problematisches Unterfangen: Unterstellt eine derartige Untersuchung doch implizit, daß es gewissermaßen derselbe Pašukanis - als Wissenschartler und Theoretiker - sei, der die in der Tar für die marxistische Rechtscheorie fundamentale "Allgemeine Recheslehre und Marxismus* geschrieben hat wic auch die hier wieder abgedruckten propagandistischen Platrirüden des Scalinismus. Damit würde aber der hier skizzierte Funktionswandel von öffentlicher Reflexion über soziale Konflikte, Strukturen und politische Herrschaftsformen und damit von Gesellschafts- und Rechrswissenschaft im Übergang zum Stalinismus, der auch jeden Theoreciker seiner individuellen Besonderheir beraubre und auf das Allgemeine eines propagandiscischen Apologeren zurechtstutzte, übersehen. Eine Kontinuität im Werk von Pašukanis, so ließe sich vorausschickend vermuten, wäre daher allenfalls zufällig.

Gleichwohl soll diese Frage hier erneut aufgegriffen werden, nicht nur deshalb, weil sie in der "Kricischen Justiz" bereits Gegenstand der Untersuchung von Norbert Reich" war, dessen Thesen hierzu problematisier werden sollen, sondern vor allem, weil die Rechrsformanalyse der "Allgemeinen Rechtslehre« zwar in diametralem Gegensatz zum stalinistischen Rechrsverständnis steht, aber dennoch von politischen Intentionen getragen ist, die seine politische Wende zun Sralinismus als folgerichrig erscheinen lassen könnten. Daß Pašukanis in der Konsequenz dieser Wende erst das Opfer seiner früheren wissenschaftlichen Einsichten brachre und

33 N. Reich, Marxisusche Rechustheoric zwischen Revolucion und Stalinismus, Das Beispiel Paschukanis, KJ $2 / 1972$ S. 194 ff. 
dann selbst als Person geopfert wurde, dies bestätigt einmal mehr, wie sehr die Diskrepanz zwischen sheoretischer Erkenntris in revolutionär-utopischer Absicht und realer geselischaftlicher Entwicklung der Sowjecunion auch die radikalsten Intellektuellen buchstäblich erst um den Verstand, dann um ihre gesellschafuliche Funktion und schließlich sogar ihre Exiscenz bringen konnte.

Norbert Reich kommt in seiner Uncersuchung zu dem Ergebnis, daß durchaus eine Kontinuitäc zwischen der *Allgemeinen Rechtslehre von Pašukanis und seinen späteren Schriften besteht: "Die Dialektik seiner eigenen Rechtstheorie will ese, so seine These, ״daß hier bereits die Grundpositionen der späteren stalinistischen Wendung in der sowjetischen Rechtstheorie ausgeformt sind ${ }^{34}$ Sehen wir uns hierzu die Rechtstheorie von Pašukanis näher an. Die politisch-theoretische Ausgangsfragestellung seiner Rechtscheorie ist die nach dem Charakter rechclicher Regelungen nach der Oktoberrevolurion, die Frage, wie die Rechtsordnung der intendierzen Ubergangsgesellschaft zum Sozialismus einzuschätzen sei. Seine Antwort ist die gegenüber allen zeitgenössischen Rechtstheoretikern radikalste: Es handele sich um eine bürgerliche Rechrsordnung, wie auch der Staat der Sowjetunion als bürgerlicher Staat anzusehen sei.

$\mathrm{Zu}$ dieser Konsequeaz wird er auf Grund seiner, wie Karl Korsch"s schreibr, "allzu orrhodoxen', abstrakten und dogmatischen Form * seiner in durchgängiger ParalleLisierung zur Marxschen Kritik der Politischen Okonomie entfalteten Argumentation gezwungen. In Auseinanderserzung mit der in der damaligen Sowjeturion verbreiteten Vorstellung von einer durch den nunmehr proletarischen Klasseninhalt ermöglichten "revolutionären Rolle des Rechts" in der Phase des Ubergangs zun Sozialismus seczr Pašukanis analog der Ausgangsfragestellung von Marx im Kapical mit der Frage an, warum die Beziehungen zwischen Menschen, warum dieser soziale Inhalt eben die Form von Rechisverhältnissen annimmt. Damit unternimmt er nach Marx als erster Theoretiker den Versuch nichc nur einer ideologiekricischen Inhalcsanalyse des Rechts oder einer Rekonstruktion seiner historischen Entwicklungsformen, sondern einer ersten systematischen Ableitung der Rechtsform als im Rechtssyscem verdinglichter, vom unmitrelbaren Eriahrungs- und Lebenszusammenhang der Individuen abstrahierter Form des sozialen Systems. Da sich nach seiner Theorie die Rechrsform unmittelbar (und ausschließlich) aus dem ökonomischen Verhälenis des Warenaustauschs, dem Austausch von äquivalenten Werten, ergibr, gelangt er konsequent zu der Schlußfolgerung, daß die entwickelsste und universelle Entfalrung der Rechtsförmigkeit aller Sozialbeziehungen spezifisches Charakteristikum der bürgerlichen Gesellschaft ist. Folgerichtig kann es in sich sozialistisch organisierenden Gesellschaften Rechtsverhältnisse nur insofern geben, wie es das Phänomen des äquivalenten Tauschs gibr: "Eine Gesellschart, die durch den Stand ihrer Produktivkräfte gezwungen ist, ein Äquivalencverhälnnis zwischen Arbeitsverausgabung und Vergürung in einer Form beizubehalten, die auch nur entfernt an den Austausch von Warenwerten erinnert, wird gezwungen sein, auch die Rechtsform beizubehalten "16. Für seine daraus sich ergebende Schlußfolgerung, daß der Charakter des Rechts in der "gegenwärig von uns erlebre(n) sneve(n), ökonomische(n) Policike «", in der Marktverhältnisse und Privatrecht rveitgehend wieder etabliert

3s K. Korsch, Besprechung von E. Pasukanis, Allgemeine Recheslehre und Marxismus, sowie von Karl Renner, Dic Rechtsinstitute des Privatrechts und iarc soziale Funtrion, ir: Archiv für die Geschichte des Sozialismus und der Arbeiterbewegung, 1930; wiederabgedruckt An Stelle einer Einleitunge in der Neuherausgabe der „Allgemeinen Rechtslehre... von Paśukanis, Fím 1966 S. I If., hier S. II. 36 Pašukanis, Allgemeine Rechsslebre..., a. a. O. S. 36 . 37 Dg., 3. a. O. S. 34 .
} 
worden waren, bürgerlich (und eben nichr sozialistische) sei, beruft er sich auf Lenin: "Das bürgerliche Recht auf dem Gebiet der Verteilung der Konsumtionsmittel setzr natürlich auch deo bürgerlichen Staat voraus ... Es ergibr sich also, daß nicht nur unter dem Kommunismus das bürgerliche Recht eine gewisse Zeit bestehen bleibr, sondern sogar der bürgerliche Staar - ohne Bourgeoisie! ${ }^{38}$

Schon aus dieser Skizze seiner Argumentation wird ersichzlich, wie sehr Pašukanis' Theorie - bei aller Fragwürdigkeir seines Ableitungsversuchs der Rechosform ${ }^{39}$ - all denen ein Dorn im Auge sein mußte, die ihr sozialiscisches Gewissen ungeachcet der fortexistierenden Rechtsformen mir definirorischen Tricks zu besänftigen suchten. So steht es für ithn außer Zweifel, daß das sowjerische Strafgesezzbuch von 1922, welches auf den Begriff der Schuld verzichtet, sowie die "Grundsätze der Strafgesetzgebung der Union* vom Zentralexekutivkomitee (ebenfalls von 1922), die die Kategorie der Strafe durch die Bezeichnung "gerichtlich-korrekcive Maßnahmen der sozialen Verteidigung" ersetzt haben, als bloße "Änderung der Terminologie weitgehend eine rein formale Reform bleiben ${ }^{40}$. Seine Kritik formuliert immer wieder unmißverständlich die revolurionär-utopische Hoffnung auf den Ubergang zu einer Gesellschaft frei assoziierter Individuen, in der alle verdinglichten, vom unmitrelbaren Lebens- und Kommunikationszusammenhang der Menschen abstrahierten und sich ihnen gegenüber verselbscändigenden Formen des sozialen Prozesses »absterbena: "Das Strafrechr, wie das Recht überhaupt, ist eine Form des Verkehrs zwischen egoistischen isolierten Subjekten, den Trägern eines autonomen Privatinteresses oder ideellen Eigentümern ... Die Begriffe des Verbrechens und der Strafe sind, wie dies aus dem Vorhergesagten klar hervorgeht, norwendige Bestimunungen der Rechrsform, von denen man sich erst wird befreien können, wenn das Absterben des rechulichen Uberbaus überhaupt anfängt. Und wenn wir in Wirklichkeir und nicht nur in Deklarationen diese Begriffe zu überwinden und ohne sie auszukommen beginnen, wird dies das beste Zeichen dafür sein, daß der enge bürgerliche Rechrshorizont sich endlich vor uns erweirert. " ${ }^{41}$ "Die Ergreifung der politischen Mache durch das Proletariat w ist für ihn zwar wdie grundlegende Voraussetzung des Sozialismus " ${ }^{42}$, jedoch ist die "Uberwindung der Rechtsform nicht nur an ein Hinausgehen über den Rahmen der bürgerlichen Gesellschaft gebunden ..., sondern auch an eine radikale Emanzipation von allen ihren Überbleibseln $\alpha^{43}$. In einer derarigen Gesellschaft, in der alles "auf den Menschen selbsi reduziert iss « ${ }^{4}$, werden "Moral, Rechr und Staat* als "Formen der bürgerlichen Gesellschaft «"s aufgehörr haben zu existieren. Ein "proletarisches Reche $\alpha$, einen proletarischen Siaat oder eine prolecarische Moral wird und kann es nach Pašukanis aber wegen der bestimmten historischen Bedingtheit der Rechrsform nicht geben: "Das Absterben gewisser Kategorien... des bürgerlichen Reches bedeutet keineswegs ihre Erset-

$38 \mathrm{Dgl}$., a. a. O.S. 35 nach Lenin, Staat und Revolucion, Wien-Berlin 1929, S. 96

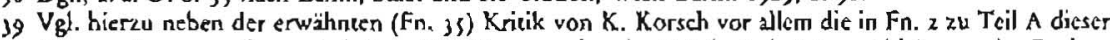
Unrersuchung erwàhnten Arbeiten. Dic Haupeaspekte der Kritik sind: Seine oAbleirung* der Rechrsform allcin aus der Warenzirkulacion (Korsch, Reich, Negr), seine Parallelisierung von Werriorm und Rechesform, dic letzrere als ein Phänomen der gesellschaitlichen Basis (und nicht des -juristischen Uberbausa) begreife (Korsch, Reich), scine Verengung des Rechisbegrill's auf das Rechr warentauschen. der Gesellschaften, auf Vereagsrecht (Rosenbaum, Negr), seine mangelnde begriffliche wie historische Unterscheidung der Konstitutionsbedingungen einer Allgemeinen Rechtslehre von denen der Rechislorm (Rosenbaum).

40 Pasǔkanis, Allgemeine Rechrslehre ..., a. a. O. S. 17.

4 I Dgl., 2. 1. O.S. 173 f.

42 Dgl, a. a. O. S. 110.

43 Dgl., a. a. O. S. 37 .

4 Dgl, a. 2 O. S. 141 .

4) Dgh, 2 a. O. S. 142 . 
zung durch neue Kategorien des proletarischen Rechts, genauso wie das Absterben der Kategorien des Wertes, Kapitals, Profits usw. bei dem Ubergang zum entfaiteten Sozialismus nicht das Auftauchen neuer proletarischer Kategorien des Werts, Kapitals usw. bedeutet ${ }^{46}$. Eindringlich expliziert er diese Vision des Untergehens aller äußerlich-abstrakten Zwangsmechanismen aus den Beziehungen der Menschen untereinander an der logisch-kategorialen Unmöglichkeit, von »sozialistischer Moralu zu reden: „Wenn das lebendige Band, das das Individuum mit der Klasse verbindet, catsächlich so stark isc, daß die Grenzen des Ichs sozusagen verwischt werden, und der Vorteil der Klasse tatsächlich mit dem persönlichen Vorteil identisch wird, dann hat es keinen Sinn mehr, von der Erfüllung einer moralischen Pflicht zu reden, dann fehlt überhaupr das Phänomen der Moral. Wo aber eine solche Verschmelzung nicht stattgefunden hat, taucht unvermeidlich das abstrakte Verhältnis der moralischen Pflicht mit allen daraus entstehenden Formen auf. Die Regel: shandle so, daß du deiner Klasse den größrmöglichen Nutzen bringste, wird der Formel Kants ganz gleich klingen: handle so, daß die Maxime deines Willens jederzeit zugleich als Prinzip einer allgemeinen Gesetzgebung gelten könnera. 47

Diese in ihrer radikalen Konsequenz vernichtende Kritik an allen Vorstellungen, denen zufolge das Weiterbestehen, ja die »Stärkung* von Recht und Staat mit dem Begriff und Ziel des Sozialismus zu idencifizieren sei, hat Pašukanis schon früh den Vorrurf des "Rechesnihilismus* eingebracht, ein Vorwurf, der bis heuce auch all diejenigen trifft, die - von Karl Korsch bis Oskar Negt $4^{4}-2 w a r$ seinen ahistorischen und einseitig an das Phänomen der Warenzirkulation fixierten Rechtsbegriff kritisieren, aber sich mit ihm in der (vielleicht utopischen) Perspekcive einer Aufhebung aller entfremderen Vergesellschaftungsformen (wie Wert, Geld, Kapital, Recht, Staat etc.) verbunden wissen - und deren Existenz zum Prüfstein für den sreal

48 So fälle Negi's Aulsatz *Thesen zur marxistischen Rechushcorie*, KJ 1973, S. I ff. Perer Römer zufolge * in eine rechesnihilistische Posicion zurück a (Recheliche Grundlagen der Politik. Zur Bedeuzung einer politischen Recheslehre, in: Demokratie und Recht H. J/1973 S. 212). Wenn auch dieser Vororurf meist mi fundamentalen Mißverständnissen des Einmaleins des historischen und dialekrischen Materialismus zu begninden versucht wird (so Römer, a. A. O. Fn. 9), scincr Intention nach zielt er auf ein nur notdüricig wrissenschaflich verbrämtes politisches Bekennenis zum - seit Stalin - offiziellen Rechtsverständnis der *soxialistischen * Länder. So folgr für Römer Negt's angeblicher Rechesnihilismus jenseits der Auseinandersetzung um die korrekec Interpretacion der Marxschen Poliöschen Okonomie aus der „Bemerkung von Negr, a. a. O., S. s, der Rechesferischismus wende aul dic Spirze getricben, wenn in der Rechestheorie der DDR die aktiv schöplerische Rolle des rechrlich poliuischen Uberbaus, cinschlicßlich der Rechtswissenschafe, berausgestellt wirde (a. 2. O. S. 235, Fn. 10). Wenn Römer ferner bedauernd konstatiert, daß in Kreisen krivischer Juristen manxiscische Rechtscheorie und Rechtstheorie von Pasukanis weiggchend gleichgeserzr werden * (a. a. O. Fn. g), wobci er dic durchweg kriuische Auseinanderselzung von $\mathrm{N}_{\mathrm{Cg}}$ mit Pasukssis olfenbar übersiehr, so ist dies nicht nur theoretisch unzutreffend, sondern ein Argumentacionsmuster, dessen Funktion in dom Versuch besteht, einen =Srigmacisierungstransfer* zu enjelen: Die seic Anfung 1937 zum stadelichen Vorurzeil geronnene Ausgrenzung von Pašukanis wird ungeachret seines Schicksals aflirmauv aufgenommen, um damit in akruellen Auseinandersetzungen andere theorctische Positionen - als mir dem gleichen Mlakel behafuer - zu diskreditieren. Dies mag mit ein Grund für die Scharfe sein, mit der Negt in der erweirerten Fassung der w 10 Thesen zur manxiscischer Rechustheorie « (in: H. Routleuthner, Rrobleme der marxistischen Recheschcoric, a. 20. S. 69 Fn. 42) auf Römer replizier har. Auch Hermann Kenncr, einer der führenden Rechtstheoretiker der DDR, enträl der peinlichen Versuchung nichr, Pasukanis als den ideologiscben Lehnneisrer von Negr anzuführen, uan sciner Kriuik an Negt historische Ticfenschärfe zu verleihen: vJede Enúconung vom Materialismus in der politischen Theorie führe aber unweigerlich dazu, dem objekriven Gang der Dinge scine Wunschvorsicllungen zu unterlegen, was bei nach links Drängenden of genug zu anarchisuischen Konsequenzen, objekziv also zu Hillsdiensen für die Reaktion, führr. Iro Falle Oskar Negus, der die von Pašukanis cntwickclte (spärer von ihm wieder aufgegebene (!)) Austauschkonzeption des Rechus aul die Arbeitskrafe verenge ...., bogründet diese scheinrevolurionäre These vor allem seine Abneigung gegen das Rechr und die Rechestheorie der Länder des rcalen Sozialismus* (Annelies und Hcrmann Klenner. Plädoyer für eine materialistische Rechustheorie, in: J. Y. Hagen, P. Römer, W. Seiffere (Hrsg.), Recheswissenschadt und Arbeiurbewegung. Festachrift für Eduard Raboísky, Köln 1976 S. 27 H., 33; Hervorhebung von mir, T. B.).
} 
existierenden Sozialismus erheben. "Wo die Rechrsform «, so resumiert Negt seine Auseinanderserzung mir Pašukanis, »und alle mit dieser verbundenen $Z$ wangsappararuren - Gerichte, Gefängnisse, Polizei, Geheimdienste, Zensur usw. - zur Regulienng des gesellschafulichen Zusammenlebens erforderlich sind, da kann dies als ein sicheres Zeichen dafür genommen werden, daß Arbeit noch nicht als erstes Lebensbedürfnis der Menschen gilc, Arbeitszwang also existiert, daß der gesellschaftliche Konstitutionszusammenbang wesentlich auf der Realabstraktion des Wertes beruht. ${ }^{49}$ Die von Pašukanis in seiner Rechtstheorie aufgeworfene Problemstellung ist daher ebensoviel und ebensowenig erledigt wie die Perspekrive einer sozialistischen Gesellschaft insgesamt.

VII.

Was verbindet nun, so bleibc zu fragen, diese Ubberlegungen des frühen Pašukanis mit seinen späteren - wie z. B. auch den hier wieder nachgedrucksen - Äußerungen? Meines Erachtens läßt sich eine solche Brücke aus dem Zusammenhang seiner rechtstheoretischen Reflexionen in keiner Weise schlagen. Tatsächlich demenciert Norbert Reich denn auch in seiner Detailuntersuchung der späteren Schriften von Pašukaris beständig seine zunächst aufgestelite Behauptung von einer in den "Grundpositionen der Allgemeinen Rechislehre bereits angelegten, ja sausgeformtena späteren stalinistischen Wende. So spricht er von einem »radikalen Kurswechsel, der in der Geschichte der Neuzeit seinesgleichen har (sucht?, T. B.) wso, von "Kurskorrektur «," dem *Zurücknehmen eigener Positionen "s2, von "Rückzugsgefechre $(n)$ " " bis hin zum *Widerruf « der $* A$ bsterbetheorie und seine(r) Behauptung, daß das Sowjetrecht keine besondere Kategorie sei«. ${ }^{34}$ Deutlicher kann die Nichtidentität seiner ursprünglichen rechrscheoretischen Bestimmung des Verhältnisses von Sozialismus und Recht mit seiner späteren stalinistischen Position kaum zum Ausdruck gebrache werden. Zuzugeben isc allerdings, daß die Wende von Pašukanis zum Stalinismus, die zur Verkehrung seiner Theorie in ihr Gegenteil führ, aus seinen gesellschatesstrukrurellen Uberlegungen zur Organisation der "Ubergangsgesellschafra und damit zur Beschleunigung des rechtlichen Absterbeprozesses nicht ohne innere Logik ist. So bestehe kein Zweifel daran, daß er jeglicher zivil- und wirtschaftsrechtlicher Kodifikation gegenüber skeprisch, ja feindlich eingestellt war - dies spricht er auch offen aus -, so daß er mit gewissern Recht als Repräsentant der linken Opposition während der Phase der "Neuen ökonomischen Politik " bezeichnet werden kann. Er erwartete, daß mit dem Aufbau einer seinheitlichen planmäßigen Wirtschafte eine ugesellschaftliche Verbindung 2 wischen den Produktionseinheiten $\alpha$ sin vernünfriger, unmaskierter Form (d. h. nicht in Warenform) ${ }^{3 s}$ entstehen werde, weil - und darin zeigt sich seine zirkulationscheoresische Reduktion der Genesis der Waren- und der Wertorm - die staatlichen Unternehmungen dann nicht mehr den Bedingungen der Zirkulation, den Marktmechanismen unterworfen wären. Forcierte Kollektivierung und die Etablierung einer umfassenden Planwirtschaft, dies sind nach seiner Konzeption die zentralen Voraussetzungen für das

49 O. Ncgr, 10 Thesen ... . , in: H. Rouleuthner (Hrsg.), aै. a. O. S. 28.

so Reich, Marxisrische Rechrsdicorie...., a. a. O. S. 162

sı Dgi., 2. a. O. S. 160 .

52 Ebd., S. 160.

1) Ebd., 5. 161.

Sf Ebd., S. 161

ss Pašukanis, Allgemcine Rechtslchre, S. 11 r. 
"allmähliche Absterben der Rechtsform überhaupta."6 Vectehlt wäre es jedoch, in dieser Vorstellung der Ersetzung von abstrakten Zwangsregelungen, die der Staat mit Hilfe von Gesezzen gewaltsam durchsetzen kann, durch rechnische Regeln des Zusammenlebens, die skonkrer (sind) und fortwährend (wechsein) nach Maßgabe der veränderten Bedingungen " $"$ ", bereits den - noch unentfalceten - Theoretiker des Scalinismus zu wähnen. Gewiß hat er ebenso wie Lenin die Möglichkeit der Entfesselung und Verselbständigung der Staatsgewait unterschätzt: Durch die Aufhebung der im System subjekriver Privatrechte zugleich auch gefesselten staatichen Interventionsmöglichkeiten zugunsten von administraciv-technischen, konkrezen Maßnahmen, deren Zusammenfassung zum einheitlichen Plan, die Monopolisierung von Entscheidung und Kontrolle beim Staat wird dieser virtuell zur einzigen und ausschließlichen Koordinationsinstanz der Gesellschaf, zum konkreten und realen Organisationszusammenhang des gesamcgesellschaftlichen Reproduktionsprozesses. Daß damit die Differenz von "Uberbau « und "Basis" keineswegs aufgehoben isc, die Funktionen und Strukruren des sozialen Zusammenhangs keineswegs in die auronome Selbsträrigkeit und Selbstverwaltung der Individuen zurückgenommen sind, hat Pasukanis zwar gesehen: "Man kann freilich darauf " (auf das Absterben der Rechtsform im Ubergang zur Planwirtschaf() "erwidern, daß z. B. ein Produkcionsprogramm auch eine öffentlich-rechtliche Norm ist, da es von der Staacsgewalt ausgeht, bindende Kraft besiczr, Rechte und Pflichten schalft usw. Freilich werden, solange die neue Gesellschafic sich aus Elementen der alten aufbaut, d.h. aus Menschen, die das gesellschaftliche Band nur als Mittel für ihre privaten Zwecke auffassen, auch die einfachen vernunfüäßigen technischen Anweisungen die Form einer dem Menschen fremden und über ihm stehenden Gewalt annehmen müssen. s $^{\S}$ Die Srunde der wendgülcigen Befreiung * erwartet er, sobald wdas Schächerverhältris und die Schächerpsychologie in der Produkcionssphäre überwunden sein wird $\$ 9$. Diese Formulierungen machen deurlich, daß er jedoch der auch unter der Bedingung der Planwirtschaft weiterbestehenden Differenz von Staat und Gesellschaft, von Abstrakcem und Konkretem keine wwesenslogische strukrurelle Bedeutung mehr zumißr. Weil er diesen technisch-administrariven Regeln die Qualiät von Rechtsnormen - seiner Ableitung der Rechtsform gemäß - nicht zuschreiben kann, reduziert sich nach ihm die gleichwohl noch fortdauemde Enctremdung auf subjektive Momente, die noch nicht überwundene privacegoistische Mencalität der Bevölkerung. Damit begibt cr sich der theorerisch begründeten Möglichkeit einer systemarischen Kricik der politischen und sozialen Mechanismen, die im Prozeß der Kollektivierung und der planmäßigen staatlichen Organisation der Produkcion als historisch neue entstehen. Angesichts dieses Defizits einer kritischen Theorie der "Ubergangsgesellschaft ", seines Vertrauens aut den konrinuierlichen evolutionären Ubergang zur kommuniscischen Gesellschalt, wird, wie Karl Korsch pointiert anmerkt, "seine ganze Becrachtungsweise unvermeidlich illusionär, da sie die gegenwärtigen Verhältnisse und Entwicklungstendenzen in Sowjetrußland nicht materiabistisch nach ihrem wirklichen Sein, sondern idealistisch nach einem ihnen subjekciv gesetzren Zweck zu begreifen suchi ${ }^{\text {to }}$. Bei aller Radikalicät und Konsequenz seiner

\footnotetext{
s6 Dol., a. a. O. S. irs.

s7 Ebd., S. IIIf.

¿8 Ebd., S. 112.

s9 Ebd., S. 112.

6o A. a. O. S. X; Frcilich kann dies Defizie einer allgemeinen Theorie der wUbergangsgescllschalta, über deren Enrwicklungasgesetze und sozialen Strukturen crst jüngst nach der Veröffentlichung von Rudolf Bahnos Dic Altemarive. Zur Kritik des real exiseicrenden Sozialismus $\alpha$, Köln, Ffm 1977 die Diskussion erneur vehement aufgeflammt ist, schwerlich dem zeitzenossischen und in diesem Entvicklungsprozeß politisch-cheorevisch engagienen Pašukanis in spezifischer Weise vorgehaken werden. Olfenbar teilt
} 
Analyse und Kricik der Rechesform als bürgerlicher - gerade auch in bezug auf die nach der Revolution fortbestehenden Rechrsverhältnisse - läßt seine Rechtscheorie gleichwohl die Möglichkeit (aber auch nur diese!) eines unkritischen Bezugs auf die Organisationsformen des sozialen Zusammenhangs und die politischen Herrschaftsstrukturen nach der Periode der "Neuen ökonomischen Politika zu. Von einer Kontinuität seiner rechtscheorecischen Positionen kann indes keine Rede sein: Von seiner *Absterbetheorie "führt kein Weg zur Propaganda des stalinistischen wstarken Staates « und seiner wsozialistischen Geseczlichkeit $\propto$

VIII.

Dies soil abschließend noch in einer knappen Skizze des Rechtsverständnisses verdeutlicht werden, welches sich unter dem Stalinismus offiziell durchserze und mir bis beute für die sich als sozialistisch bezeichnenden Länder (aber auch für diejenigen politischen Organisationen, die solche geselischaftlichen Verhälnisse sich zum Vorbild nehmen) charakteristisch zu sein scheint. Das seit dem Stalinismus dominierende Rechtsverständnis, wie es vor allem von Vys̆inskij, dem Staarsankläger der Moskzauer Prozesse formuliert wurde ${ }^{61}$, wird häufig als "positiviscisch $\alpha^{62}$ und "normarivistisches bezeichnet. Damit wird die unhinterfragte Geltung des Rechrs kraft staaclicher Setzung und zugleich sein ahistorischer, geschichrsunabhängiger Geltungsanspruch bezeichnet. Dieses positivistische Rechtsverständnis gehe einher mit einem »idealistischeo Voluncarismus ${ }^{64}$, weil dem Recht als willenclicher normativer Setzung eine aktive Rolle bei der Gestaltung und Veränderung der gesellschaftlichen Verhälınisse zugeschrieben wird. So gewiß diese Charakterisierung sich mir zahlreichen Äußerungen aus der damaligen Zeir über das w Wesen des Rechts» belegen läßr, so wenig trifft sie jedoch $\mathrm{m}$. E. den zentralen Punkt, den dieses Rechtsverständnis von bürgerlichen Varianten des positivistischen Normativismus unterscheidet. Am treffendsten erscheint für dieses Rechtsverständnis, wie es sehr deutlich in der Präsentation der Stalinverfassung durch Pašukanis zum Ausỏruck

aucb Negt die-m. E. zu problemavisierende - Vorstellung von einer wersten Phase des Kommunismusa, in der dic . die Rechisverhältnisse staatlicb sichernden Normen durch gewohnheitsmäßige Regelna ensetzr werden (10 Thesen..., a. a. O. S. 17), öffentliche Funkzionen in einfachz und übersichtliche administrative Funktionen verwandelt werden.

61 A. J. Vyšinskij, Zur Lage an der theoretischen Rechesfront, 1937. auszugsweise nachgedruckt in: $N$ Reich (Hrsg.), Marxissische und sozialisciscbe Rechesthearic, S. 11 ff. In dieser Schrif geht Vyšinskij in ähnlich grober Weise mir Pasukanis ins Gericht - und legirimien damit dessen Liquidienung - wie Pašukanis in dem ersten, hier vieder nachgedruckten Aufsatz mit anderen opposicionellen (bzw. als solche bezeichneten) Positionen. Hier finder sich auch explizir der Vorwur des $*$ Rechtsnihilismus $x$ ${ }_{-}$Die Schädlingsarbeit im Bereich der Rechisdisziplinen haben Pasukanis und seinc Handlanger geschickt angestellt. Mehrere Male haben sie ihre Fehler anerkannt, jedoch, wre jetzr festgestellt wurde, nur deshalb. um ihre scheinmarxistischen, antileninistischen Ansichen in einer versteckten Form fortzuführen. ... Den Siudenten und Kadern brachteted sie eine nihilistische Beziehung zum Sowjetrechr bei (1. 2 O. S. 114). Später, rach dem Ende der ersten großen Säuberungswelle und dem Abschluß des zweiren Weltkrieges hat Vysunskij seine $*$ Rachtsthearic* in der Schrift $*$ Die Lehre Lenins-Stalins von der proletarixchen Revolucion und vom Staat., Berlin 1949, zusammengefaßr.

$62 \mathrm{Ncert,} \mathrm{2.} \mathrm{2.} \mathrm{O.S.} 23$ charakıcrisien Vyšinskij als einen der scharfsinnigsten Theoretiker der positivistisch geprägren manxistischen Rechtslehre*. Von Scharfsinn kann allerdings in dessen Arbeicen m. E. ebenso wenig die Rede sein wic in den hier wiader publizicren Studien von Pašukanis: Es sind propagandisische Legicimationsschríften, die das Manko kuger Gedanken durch eine sich stark gerierende Dikion ersezzen. Ebenso wie Negr sprich, Klaus Westen. (Die rechustheoretischen und rechtspolirischen Ansichten Josef Stalins, Lindau und Konstanz : 959 ) von der Enrwicklung des sowjeriscben Siaztes zu einem uposivivisuischen Geserzesstaata (a. a. O. S. 96).

6) Klaus Westen, a. a. O.S. 87 ; Negt, 2. 2. O.S. 24; N. Reich, Sozialismus und Zivilrecht, a. 2. O. S. 265. 273.

64 Negr, a. 2. O. S. 10 
komma, die Bezeichnung als snaiver Begriffsrealismus $\alpha$, womit derselbe Wahrnehmungs- und Reflexionsmechanismus angesprochen werden soll, der auch die gleichsam magische Sprachweise der Propaganda insgesamt bescimmt und diese Kommunikationsform als vorbürgerlich erscheinen läßr. Dieser nnaive Begriffsrealismus* differenzier nicht zwischen Begriff und Realicät, zwischen Symbol und symbolisiertem Gegenstand, zwischen Recht und sozialer Wirklichkeit. Das System ist sozialistisch, weil es die Verfassung so besagt, und weil es die Verfassung so sagt, stärkt dies den real existierenden Sozialismus. Und weil der nomentlaturhafi ins Leben gesetzte "Sozialismus etwas schlechthin Positives darstellt, in diesem einfachen und klaren Begriff aber immer der Realizät (mit ihren schlechten Widersprüchen) vorauseilt (also ihr idealisiertes Sein meint), deshalb muß sich jeder beständig auf den Boden dieser Formel stellen, sich auf sie beziehen. Kritik erwa an der Formulierung der Verfassung vom sozialiscischen Staat * würde die "Realität* leugnen, was entweder auf der Basis der naiven Widerspiegelungspsychologie als krankhafce Wahrnehmungsstörung anzusehen wäre (mir der Konsequenz: Irrenhaus für Dissidenten) oder aber als Unterlassung der Stärkung des Sozialismus ausgelegt würde, weil der Kritiker nicht positiv durch den Gebrauch des Worres "sozialistisch « daran mitarbeitet, daß die Realität mit ihrem im Symbol idealisierten Sein vollends zur Übereinscimmung kommt (er wäre ein Feind des Sozialismus).

Dieser magische (in hiscorisch-politischen Kategorien: feudale) Begrifisrealismus ist keineswegs mit dem bürgerlichen Rechtspositivismus vergleichbar. ${ }^{6+2}$ Denn der juristische Positivismus war sich immer darüber im klaren, daß es sich um einen normativen Positivismus handele, nicht um empirische Realitätsaussagen. Ihm ging es um logisch geschlossene, möglichst "normidentische « Ableitung von Normen aus höheren Normen und obersten Grundnormen, um exakse Begriffsbestimmung und klare methodisch operationalisierbare Subsumtionsregeln. An all dem fehlt es beim sowjetischen Begriffsrealismus, der der mictelalterlichen Hochscholasrik eines Thomas v. Aquin sehr viel näher steht. Weil die Norm die Realität nicht bloß darstellt, sondern ist, gibt es so etwas wie einen Subsumtionsvorgang überhaupe richt, der ja immerhin die Möglichkeit impliziert, daß ein Sachverhalt nicht unter den Begriff zu bringen ist. Da aber die sowjetische Gesellschaft insgesamt auf die Realicät der einfachen und idencischen Begriffe wie »sozialistisches Eigentum", "sozialistische Arbeitsformen«, „demokratischste Macht der Welt«, "Werktätige* gebracht ist, existiert eine Abweichung vom Begriff schon der theorecischen Möglichkeit nach nicht.

Damit wird aber Recht, wird die Verfassung selbst zu einer weiteren Form des herrschenden symbolischen Kommunikaxionsmechanismus: Der Propaganda. Daneben enthält sie rechnische Organisarionsregehn. Diese rein propagandistische Form verkennt gründlich, wer meint, er könne sich etwa aut den Grundrechtsteil der stalinistischen Verfassung berufen - gegen den Sraac. Das wäre nach diesem Rechesverständnis konterrevolutionär, weil doch allein die Tatsache, daß diese Grundrechte in der Verfassung verankert sind, besagt, daß sie auch in der Realität

64a Die Ausführungen im folgenden zielen auf die Differenz des jeweiligen Rechtsverständrisses sowie der daraus begnindezen juristischen Methoden. Eine vergleichende Untersuchung der Fumkewanen des bürgerlichen siaazrechtichen Posievismus (vgl. Peter v. Oertzen. Die soziale Funterion des staatsrechtlichen Positivismus, Ffm 1974) mil dem rtaxlssozialisuschen ensiven Begriffsrcalismus" steht noch aus:

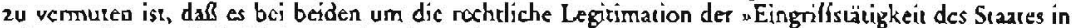
nahezu alle gesellschafilichen Lebensbereichea (D. Secrel, Nacbwor ebd., S. 3S4) gehi, Jedoch unterscheiden sich mit der grundlegenden Diskrepanz der jeweiligen Vergescllschaftungsmuster sowobl dic Formen seaalichen Handelns als aucb ihre spezifischen Leginimationsprobleme deran fundamental, daß auch auf dieser Ebene der funkrionalen Analyse die Differemzen im Vordergrund stehen. 
der Gesellschaft entfater sind und bestehen. Wer sie einklagt, würde dies aber leugnen.

Auch zu diesem stalinistischen Verständnis des Rechts (welches schwerlich als Rechtscheorie bezeichner werden kann), weist die wAllgemeine Rechtslehre von Pašukanis keinen plausiblen Weg. So sehr man auch mir Korsch ${ }^{65}$ den dogmatischen, ja "scholastischen" Zug seiner analog zur Marxschen Wertformanalyse der Ware entfalteren Rechrsformanalyse oder auch mit Wolf Rosenbaum ${ }^{66}$ den ahistorischen, einseitig an den römisch-rechtlichen, kontinentalen Rechtsordnungen orienrierten Versuch einer Kritik aligemeiner juristischer Grundbegriffe kricisieren mag: Seine Untersuchung der spezifischen sozialstrukturellen Voraussetzungen für die Genesis des juristischen Elements in den Beziehungen der Menschen zueinander basiert auf einem genuin materialistischen Gesellschafts- und Rechtsverständnis, welches er einleitend in seiner Rechtslehre in Auseinanderserzung gerade auch mir positivistischen und normativistischen Rechtscheorien expliziert. Die spätere Übernahme des platten begriffsrealistischen Rechtsjargons der Stalinära kann von dort ausgehend nicht als Entwicklung verstanden werden; folgerichtig wäre allenfalls das Eingeständnis einer verstärkten Rückkehr zu zunehmend verrechtlichten, $d$. h. nach seiner Theorie "bürgerlichen« Formen des sozialen Zusammenhangs getvesen oder aber die konsequent durchgehaltene Negierung des Rechrscharakters der staaclichen Regelungen. Beide Konsequenzen, so scheint es, waren aus einsehbaren politischen wie intellektuellen Gründen kaum durchzuhalcen: Die erste, weil sie die endgültige Absage an einen revolutionären Traum bedeuter härte, dessen Realisierung in scheinbar so greifbare Nähe gerückt war. Die zweite, weil sie nur gegen die allï̈gliche Erfahrung der zunehmenden Expansion rechelich geregelter Beziehungen - also gegen die herrschende Realität - durchzuhalten gewesen wäre. Den Ausweg aus diesem Dilemma, den Versuch einer Reformulierung der "Allgemeinen Rechrslehre* im Interesse einer kapitalismusübergreifenden Bestimmung der sozialen Genesis der Rechrsform und einer sysremacischen Reflexion über das Verhälnnis von Recht und Plan, von stechnischena, bürokratisch-adminisrrativen und rechdichen Regeln hac Pašukanis ebensowenig unternommen wie nach ihm irgendeiner der Rechtstheoretiker dieser ssozialistischen« Länder oder der mit ihnen scrikt lüerten "Bruderparteien*. Zu sehr war die Rechtstheorie als Teil der Gesellschaftswissenschaften schon in den Dienst der Propaganda gestellt worden, als daß sie die Spaltung in eine Kritik des Rechts bürgerlicher Gesellschaften einerseits und eine stalinistische Legitimationswissenschaft andererseits noch härte aufbrechen könnenz. ${ }^{67}$

6s A. .. O. S. X.

66 A. 3. O.S. 161 if.

67 Mariscische Rechescheorie degenericr in der Sowjexunion seir der Stalin-Xara zu dem mühseligen Unterfangen, die wachsende Diskrepanz von historisch-materialistischer, kvitischer Gesellschafuscheorie und sozialer Realität, dic sich zunächst in offensichtlichen Paradoxien ausdrückt, zu systcmatisieren und uner einen einheitlichen (udialekuischena) Begnilf zu bringen. Saljn, der in scinen Irihen Schrifen ebentalls wie selbstverstandlich von $U_{c r}$-Absterbecheorien von Staat und Recht ausging (vgl. hierzu Westen, a. a. O. S. Ir 2 fr.) bringt diese von der gesellschaldichen Entricklung der Sowjerurion beständig demenciere Vorstellung in dic nachlolgende paradoxe Formulierung: WWir sind lür das Abstcrben des Sraates. Wis sind jedoch gieichzeirig für die Verstärkung der Diktacur des Psoletariats, dic die stàrteste, mächtigste Staatsgewalt ist, die je bestandea hat. Höchste Entwicklung der Stastsmachr zur Vorbereirung der Bcdingungen für das $\Lambda$ bstcrben des Staatsmacht - so lauret die marxisusche Formela (Stalin, Der Manxismus und die nationale und koloniale Fragc, Moskau 1939. S. 280). 